\title{
Isaac Newton
}

Mathématicien, Physicien, Philosophe anglais (1643-1727)

\section{Principes mathématiques de la Philosophie naturelle}

\author{
(Philosophiae Naturalis Principia Mathematica)
}

\author{
Par feue Madame la Marquise du Chastellet \\ Paris, 1759
}

\section{Définitions.}

\section{Axiomes ou Lois du Mouvement.}

Un document produit en version numérique par Jean-Marc Simonet, bénévole, Courriel : Jean-Marc_Simonet@uqac.ca

Dans le cadre de la collection: "Les classiques des sciences sociales" Site web : $\underline{\text { http://classiques.uqac.ca/ }}$

Une collection développée en collaboration avec la Bibliothèque Paul-Émile-Boulet de l'Université du Québec à Chicoutimi Site web : http://bibliotheque.uqac.ca/ 


\section{Politique d'utilisation de la bibliothèque des Classiques}

Toute reproduction et rediffusion de nos fichiers est interdite, même avec la mention de leur provenance, sans l'autorisation formelle, écrite, du fondateur des Classiques des sciences sociales, Jean-Marie Tremblay, sociologue.

Les fichiers des Classiques des sciences sociales ne peuvent sans autorisation formelle:

- être hébergés (en fichier ou page web, en totalité ou en partie) sur un serveur autre que celui des Classiques.

- servir de base de travail à un autre fichier modifié ensuite par tout autre moyen (couleur, police, mise en page, extraits, support, etc...),

Les fichiers (.html, .doc, .pdf, .rtf, .jpg, .gif) disponibles sur le site Les Classiques des sciences sociales sont la propriété des Classiques des sciences sociales, un organisme à but non lucratif composé exclusivement de bénévoles.

Ils sont disponibles pour une utilisation intellectuelle et personnelle et, en aucun cas, commerciale. Toute utilisation à des fins commerciales des fichiers sur ce site est strictement interdite et toute rediffusion est également strictement interdite.

\section{L'accès à notre travail est libre et gratuit à tous les utilisa- teurs. C'est notre mission.}

Jean-Marie Tremblay, sociologue

Fondateur et Président-directeur général, LES CLASSIQUES DES SCIENCES SOCIALES. 
Cette édition électronique a été réalisée par Jean-Marc Simonet, ancien professeur des Universités, bénévole.

Courriel : Jean-Marc_Simonet@uqac.ca

À partir de :

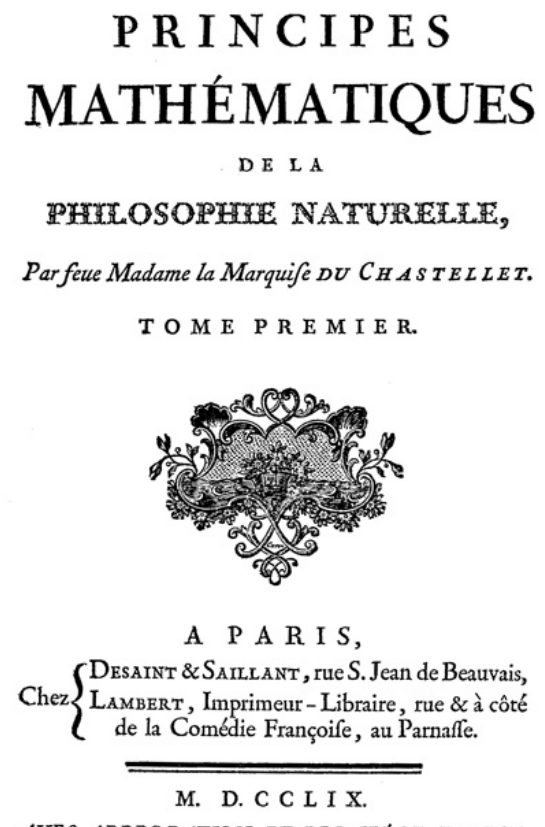

AVEC APPROBATION ET PRIVILEGE DU ROI.
Isaac Newton

Physicien, Mathématicien, Philosophe anglais

(1643-1727)

\section{Principes mathématiques de la Philosophie naturelle}

\author{
Par feue Madame la marquise \\ du Chastellet \\ chez Desaint \& Saillant et Lambert, im- \\ primeurs, Paris, 1759, \\ deux tomes de 437 p. et 379 p.
}

Polices de caractères utilisées :

Pour le texte: Times New Roman, 14 et 12 points.

Pour les notes de bas de page : Times New Roman, 10 points.

Édition électronique réalisée avec le traitement de textes Microsoft Word 2008 pour Macintosh.

Mise en page sur papier format : LETTRE (US letter), 8.5’’ x 11'’)

Édition numérique réalisée le 3 juin 2010 à Chicoutimi, Ville de Saguenay, province de Québec, Canada

\section{Fait avec}




\section{Table des matières}

Avertissement de l'éditeur (édition 1756)

Préface historique (de Monsieur de Voltaire)

Préface de Monsieur Newton à la première édition (1686)

Préface de Monsieur Newton à la tête de la seconde édition

Préface de Monsieur Newton à la troisième édition

Préface de M. Cotes (à la présente édition, de 1759)

Sur la physique de Newton, à Madame la Marquise du Chastelet (M. de Voltaire)

Principes mathématiques de la Philosophie Naturelle.

Définitions.

Axiomes ou Lois du Mouvement.

Du mouvement des corps. - Livre Premier.

Section I. - De la méthode des premières et dernières raisons employée dans tout cet ouvrage.

Section II. - De la recherche des forces centripètes.

Section III. - Du mouvement des corps dans les sections coniques excentriques.

Section IV. - De la détermination des orbes elliptiques, paraboliques et hyperboliques, lorsque l'un des foyers est donné.

Section $V$. - De la détermination des orbites lorsqu'aucun des foyers n'est donné.

Section VI. - De la détermination des mouvements dans des orbes donnés.

Section VII. - De l'ascension et de la descente rectiligne des corps. 
Section VIII. - De la détermination des orbes que décrivent des corps sollicités par des forces centripètes quelconques.

Section IX. - Du mouvement des corps dans des orbes mobiles, et du mouvement des apsides.

Section X. - Du mouvement des corps dans des superficies données, et des oscillations des corps suspendus par des fils.

Section XI. - Du mouvement des corps qui s'attirent mutuellement par des forces centripètes.

Section XII. - Des forces attractives des corps sphériques.

Section XIII. - Des forces attractives des corps qui ne sont pas sphériques.

Section XIV. - Du Mouvement des corpuscules attirés par toutes les parties d'un corps quelconque.

\section{Du mouvement des corps. - Livre Second.}

Section I. - Du mouvement des corps qui éprouvent une résistance en raison de leur vitesse.

Section II. - Du mouvement des corps qui éprouvent une résistance en raison doublée des vitesses.

Section III. - Du mouvement des corps qui éprouvent des résistances qui sont en partie en raison de la vitesse, et en partie en raison doublée de cette même vitesse.

Section IV. Du mouvement circulaire des corps dans les milieux résistants...

Section V. - De la densité et de la compression des fluides et de l'hydrostatique.

Section VI. - Du mouvement et de la résistance des corps oscillants.

Section VII. - Des mouvements des fluides et de la résistance des projectiles.

Section VIII. - De la propagation du mouvement dans les fluides.

Section IX. — Du mouvement circulaire des fluides.

\section{Du Système du Monde — Livre Troisième.}

Règles qu'il faut suivre dans l'étude de la physique.

Phénomènes.

Propositions.

Du mouvement des nœuds de la Lune. 


\section{Suivi du \\ Commentaire des Principes Mathématiques \\ de la Philosophie Naturelle \\ Par Madame la Marquise du Chastellet}

Exposition abrégée du Système du Monde.

Introduction contenant une histoire abrégée du développement du vrai Système de l'Univers.

Chapitre I. Principaux phénomènes du Système du Monde

Chapitre II. Comment la théorie de M. Newton explique les phénomènes des planètes principales

Chapitre III. De la détermination de la figure de la Terre, selon les principes de M. Newton

Chapitre IV. Comment M. Newton a expliqué la précession des équinoxes.

Chapitre V. Du flux et reflux de la mer

Chapitre VI. Comment M. Newton explique les phénomènes des planètes secondaires, et principalement ceux de la Lune

Chapitre VII. Des comètes

Solution analytique des principaux problèmes qui concernent le Système du Monde

Section I. - Des trajectoires dans toutes sortes d'hypothèses de pesanteur

Section II. - De l'attraction des Corps en ayant égard à leurs figures

Section III. - Explication de la réfraction de la lumière, en employant le principe de l'attraction

Section IV. - De la figure de la Terre

Section V. - Des marées 


\section{Avertissement de l’éditeur}

Cet ouvrage est composé de deux Parties. La première est une traduction du texte littéral des Principes Mathématiques de la Philosophie Naturelle. Il est presque superflu d'avertir qu'elle a été faite sur la dernière édition de 1726 , édition qui l'emporte sur toutes les précédentes par rapport aux corrections suggérées par des idées postérieures, et par les remarques de quelques célèbres mathématiciens. L'illustre interprète, plus jalouse de saisir l'esprit de l'auteur, que ses paroles, n'a pas craint en quelques endroits d'ajouter ou de transposer quelques idées pour donner au sens plus de clarté. En conséquence ou trouvera souvent Newton plus intelligible dans cette traduction que dans l'original ; et même que dans la traduction anglaise. En effet on s'est tellement attaché dans cette dernière au texte littéral de l'Auteur, que s’il y a quelque ambiguïté dans le Latin, on la retrouve dans l'Anglais. Tant de timidité donnerait lieu de soupçonner l'Auteur d'avoir faiblement entendu son original, et d'avoir usé de la ressource ordinaire en pareil cas : c'est de rendre les mots quand on ne peut rendre les choses. Nous aimons pourtant mieux penser que cette scrupuleuse fidélité vient d'un autre motif, et l'attribuer à un certain respect si justement acquis à cet immortel Ouvrage, respect qui a engagé son traducteur à le rendre trait pour trait.

À l'égard de la confiance que le Public doit avoir dans cette traduction, il suffit de dire qu'elle a été faite par feue Madame la Marquise du Châtelet, et qu'elle a été revue par $M$. Clairaut.

La seconde partie de l'Ouvrage est un Commentaire des endroits des principes, relatifs au système du monde. Ce Commentaire est luimême divisé en deux parties, dans la première desquelles on expose de la manière la plus sensible, les principaux phénomènes dépendants de l'attraction: ces découvertes jusqu'à présent hérissées de tant d'épines, seront désormais accessibles à tous les Lecteurs capables de quelque attention, et qui auront de légères notions des Mathématiques. 
À cette partie du Commentaire en succède une plus savante. On y donne par analyse la solution des plus beaux problèmes du système du monde : on y examine la forme qu'ont réellement ou qu'auraient les orbites des planètes dans les différentes hypothèses de pesanteur, l'attraction qu'exerceraient des corps de différentes figures, la réfraction de la lumière, effet de l'attraction des parties insensibles des corps, la théorie de la figure de la Terre et celle des marées. Toutes ces recherches sont tirées pour la plupart ou des Ouvrages de M. Clairaut ou des cahiers qu'il donnait en forme de leçons à M. le Comte du Châtelet Lomont, fils de l'illustre Marquise. L'avantdernière section est un excellent précis de son Traité sur la figure de la Terre. La dissertation du savant $M$. Daniel Bernoulli, qui a remporté le prix proposé pour la question des marées forme le fond de la dernière : elle est de plus augmentée de diverses notes et éclaircissements que l'auteur a communiqués.

On s'étonnera sans doute que ce Commentaire ne s'étende pas plus loin ; mais je l'ai déjà dit, son Auteur a cru devoir se borner à ce qui concerne plus particulièrement le système du monde. Dans cette vue, il n’y a pas jugé nécessaire de commenter la partie des Principes qui contient la théorie des fluides. D’ailleurs cette théorie a été traitée par tant de mains, et en particulier avec tant du succès par MM. Daniel Bernoulli et d'Alembert, dont les écrits sont entre les mains de tout le monde, qu'il devenait superflu d'y toucher. À l'égard de la théorie des Comètes, on trouve dans la première partie du Commentaire un article entier qui les concerne et qui doit suffire. La détermination géométrique de la forme de leurs orbites est contenue dans le problème général des trajectoires, et c'est dans les traités d'Astronomie qu'on doit chercher la manière d'en déterminer la forme et la position d'après les observations. M. le Monnier a suffisamment rempli cet objet dans ses éléments d'astronomie, et ceux qui ne trouveraient pas une clarté suffisante dans le texte même du troisième livre des Principes de $M$. Newton, peuvent recourir à ces éléments comme à un excellent Commentaire.

Il n'y a que la théorie des planètes secondaires dont le manque dans cet Ouvrage semblerait plus difficile à justifier ; mais au temps où $M$. Clairaut travaillait avec Madame du Châtelet, il était encore trop peu content, et de ce que Newton avait fait sur ce sujet, et de ses idées propres, pour lui en rien communiquer. Cette partie intéressante 
du système du monde n'a reçu que depuis peu cette perfection qui lui manquait. Pour suppléer à ce défaut, on doit consulter la pièce de $M$. Clairaut qui a remporté le prix de l'Académie de Petersbourg sur la théorie de la Lune, et la première partie de l'Ouvrage que M. d'Alembert vient de publier sous le titre de Recherches sur quelques points importants du système du Monde.

C'est là tout ce qu'en qualité d'éditeurs nous avons à dire de cet ouvrage. M. de Voltaire a pris la peine de tracer le caractère de la savante Dame qui en est l'auteur. La préface historique qu'on lit à la suite de cet Avertissement est de cet homme célèbre. 


\section{Préface historique}

Cette traduction que les plus savants Hommes de France devaient faire, et que les autres doivent étudier, une femme l'a entreprise et achevée à l'étonnement et à la gloire de son pays. Gabrielle-Émilie de Breteuil, Marquise du Châtelet, est l'Auteur de cette traduction, devenue nécessaire à tous ceux qui voudront acquérir ces profondes connaissances, dont le monde est redevable au grand Newton.

C’eût été beaucoup pour une femme de savoir la géométrie ordinaire, qui n'est pas même une introduction aux vérités sublimes contenues dans cet Ouvrage immortel. On sent assez qu'il fallait que Madame la Marquise du Châtelet fût entrée bien avant dans la carrière que Newton avait ouverte, et qu'elle possédât ce que ce grand homme avait enseigné. On a vu deux prodiges : l'un, que Newton ait fait cet Ouvrage ; l'autre, qu'une Dame l'ait traduit et l'ait éclairci.

Ce n'était pas son coup d'essai, elle avait auparavant donné au public une explication de la philosophie de Leibnitz sous le titre d'Institutions de Physique, adressées à son fils, auquel elle avait enseigné elle-même la Géométrie.

Le Discours préliminaire qui est à la tête de ses institutions est un chef-d'œuvre de raison et d'éloquence : elle a répandu dans le reste du livre une méthode et une clarté que Leibnitz n'eut jamais ; et dont ses idées ont besoin, soit qu'on veuille seulement les entendre, soit qu'on veuille les réfuter.

Après avoir rendu les imaginations de Leibnitz intelligibles, son esprit qui avait acquis encore de la force et de la maturité par ce travail même, comprit que cette Métaphysique si hardie, mais si peu fondée, ne méritait pas ses recherches. Son âme était faite pour le sublime, mais pour le vrai. Elle sentit que les monades et l'harmonie préétablie devaient être mises avec les trois éléments de Descartes, et que des systèmes qui n’étaient qu'ingénieux, n'étaient pas dignes de l'occuper. Ainsi, après avoir eu le courage d'embellir Leibnitz, elle 
eut celui de l'abandonner : courage bien rare dans quiconque a embrassé une opinion, mais qui ne coûta guères d'efforts à une âme qui était passionnée pour la vérité.

Défaite de tout esprit de système, elle prit pour sa règle celle de la Société Royale de Londres, Nullius in verba; et c'est parce que la bonté de son esprit l'avait rendue ennemie des partis et des systèmes, qu'elle se donna toute entière à Newton. En effet Newton ne fît jamais de système, ne supposa jamais rien, n’enseigna aucune vérité qui ne fût fondée sur la plus sublime Géométrie ou sur des expériences incontestables. Les conjectures qu'il a hasardées à la fin de son Livre sous le nom de Recherches, ne sont que des doutes, il ne les donne que pour tels ; et il serait presque impossible que celui qui n'avait jamais affirmé que des vérités évidentes, n’eût pas douté de tout le reste.

Tout ce qui est donné ici pour principe, est en effet digne de ce nom, ce sont les premiers ressorts de la nature, inconnus avant lui : et il n'est plus permis de prétendre à être Physicien sans les connaître.

Il faut donc bien se garder d'envisager ce Livre comme un système, c'est-à-dire comme un amas de probabilités qui peuvent servir à expliquer bien ou mal quelques effets de la Nature.

S'il y avait encore quelqu'un d'assez absurde pour soutenir la matière subtile et la matière cannelée, pour dire que la Terre est un Soleil encroûté, que la Lune a été entraînée dans le tourbillon de la Terre, que la matière subtile fait la pesanteur, et toutes ces autres opinions romanesques substituées à l'ignorance des Anciens, on dirait: Cet homme est Cartésien. S’il croyait aux monades, on dirait : Il est Léibnitien ; mais on ne dira pas de celui qui fait les éléments d'Euclide, qu'il est Euclidien : ni de celui qui sait d'après Galilée en quelle proportion les corps tombent, qu'il est Galiléiste. Aussi en Angleterre ceux qui ont appris le calcul infinitésimal, qui ont fait les expériences de la lumière, qui ont appris les lois de la gravitation, ne sont point appelés Newtoniens : c'est le privilège de l'erreur de donner son nom à une Secte.

Si Platon avait trouvé des vérités, il n’y eût point eu de Platoniciens, et tous les hommes auraient appris peu à peu ce que Platon avait enseigné ; mais parce que dans l'ignorance qui couvre la Terre, les uns s'attachaient à une erreur, les autres à une autre, on combattait 
sous différents étendards : il y avait des Péripatéticiens, des Platoniciens, des Épicuriens, des Zénonistes, en attendant qu'il y eût des Sages.

Si on appelle encore en France newtoniens les philosophes qui ont joint leurs connaissances à celles dont Newton a gratifié le genre humain, ce n'est que par un reste d'ignorance et de préjugé. Ceux qui savent peu et ceux qui savent mal, ce qui compose une multitude prodigieuse, s'imaginèrent que Newton n'avait fait autre chose que combattre Descartes, à peu près comme avait fait Gassendi : ils entendirent parler de ses découvertes, et ils les prirent pour un système nouveau. C'est ainsi que quand Harvée eu rendu palpable la circulation du sang, on s'éleva en France contre lui : on appela Harvéistes et Circulateurs ceux qui osaient embrasser la vérité nouvelle que le public ne prenait que pour une opinion. Il le faut avouer, toutes les découvertes nous sont venues d'ailleurs, et toutes ont été combattues. Il n’y a pas jusqu'aux expériences que Newton avait faites sur la lumière, qui n’aient essuyé parmi nous de violentes contradictions. Il n'est pas surprenant après cela que la gravitation universelle de la matière ayant été démontrée, ait été aussi combattue.

Il a fallu, pour établir en France toutes les sublimes vérités que nous devons à Newton, laisser passer la génération de ceux qui ayant vieilli dans les erreurs de Descartes, turpè putaverunt parere minoribus, et quae imberbes didicere, fenes perdenda fateri.

Madame du Châtelet a rendu un double service à la postérité en traduisant le Livre des Principes, et en l'enrichissant d'un Commentaire. Il est vrai que la Langue Latine dans laquelle il est écrit, est entendue de tous les savants; mais il en coûte toujours quelques fatigues à lire des choses abstraites dans une langue étrangère : d'ailleurs le Latin n'a pas de termes pour exprimer les vérités mathématiques et Physiques qui manquaient aux anciens.

Il a fallu que les modernes créassent des mots nouveaux pour rendre ces nouvelles idées. C’est un grand inconvénient dans les Livres de sciences, et il faut avouer que ce n'est plus guères la peine d'écrire ces Livres dans une langue morte, à laquelle il faut toujours ajouter des expressions inconnues à l'antiquité, et qui peuvent causer de l'embarras. Le Français qui est la Langue courante de l'Europe, et qui 
s'est enrichi de toutes ces expressions nouvelles et nécessaires, est beaucoup plus propre que le Latin à répandre dans le monde toutes ces connaissances nouvelles.

À l'égard du Commentaire Algébrique, c'est un ouvrage au-dessus de la traduction. Madame du Châtelet y travailla sur les idées de M. Clairaut : elle fit tous les calculs elle-même, et quand elle avait achevé un Chapitre, M. Clairaut l'examinait et le corrigeait. Ce n'est pas tout, il peut dans un travail si pénible échapper quelque méprise ; il est très aisé de substituer en écrivant un signe à un autre ; M. Clairaut faisait encore revoir par un tiers les calculs, quand ils étaient mis au net, de sorte qu'il est moralement impossible qu'il se soit glissé dans cet Ouvrage une erreur d'inattention ; et ce qui le serait du moins autant, c'est qu'un Ouvrage où $\mathrm{M}$. Clairaut a mis la main, ne fût pas excellent en son genre.

Autant qu'on doit s'étonner qu'une femme ait été capable d'une entreprise qui demandait de si grandes lumières et un travail si obstiné, autant doit-on déplorer sa perte prématurée. Elle n’avait pas encore entièrement terminé le Commentaire, lorsqu'elle prévit que la mort pouvait l'enlever ; elle était jalouse de sa gloire et n'avait point cet orgueil de la fausse modestie, qui consiste à paraître mépriser ce qu’on souhaite, et à vouloir paraître supérieure à cette gloire véritable, la seule récompense de ceux qui servent le Public, la seule digne des grandes âmes, qu'il est beau de rechercher, et qu'on n'affecte de dédaigner que quand on est incapable d'y atteindre.

Elle joignit à ce goût pour la gloire, une simplicité qui ne l'accompagne pas toujours, mais qui est souvent le fruit des études sérieuses. Jamais femme ne fut si savante qu'elle, et jamais personne ne mérita moins qu'on dise d'elle, c'est une femme savante : elle ne parlait jamais de science qu'à ceux avec qui elle croyait pouvoir s'instruire, et jamais n'en parla pour se faire remarquer. On ne la vit point rassembler de ces cercles où il se fait une guerre d'esprit, où l'on établit une espèce de tribunal, où l'on juge son siècle, par lequel, en récompense, on est jugé très sévèrement. Elle a vécu longtemps dans des sociétés où l'on ignorait ce qu'elle était, et elle ne prenait pas garde à cette ignorance. 
Née avec une éloquence singulière, cette éloquence ne se déployait que quand elle avait des objets dignes d'elle. Ces Lettres où il ne s'agit que de montrer de l'esprit, les petites finesses, ces tours délicats que l'on donne à des choses ordinaires, n'entraient point dans l’immensité de ses talents ; le mot propre, la précision, la justesse et la force étaient le caractère de son éloquence ; elle eut plutôt écrit comme Pascal et Nicole, que comme Madame de Sévigné. Mais cette fermeté sévère et cette trempe vigoureuse de son esprit ne le rendaient pas inaccessible aux beautés des sentiments : les charmes de la Poésie et de l'Éloquence la pénétraient, et jamais oreille ne fut plus sensible à l'harmonie. Elle savait par cœur les meilleurs vers, et ne pouvait souffrir les médiocres. C’était un avantage qu'elle eût sur Newton, d'unir à la profondeur de la Philosophie, le goût le plus vif et le plus délicat pour les Belles Lettres.

On ne peut que plaindre un Philosophe réduit à la sécheresse des vérités, et pour qui les beautés de l'imagination et du sentiment sont perdues.

Dès sa tendre jeunesse elle avait nourri son esprit de la lecture des bons Auteurs, en plus d'une Langue ; elle avait commencé une traduction de l'Énéide dont j'ai vu plusieurs morceaux remplis de l'âme de son Auteur : elle apprit depuis l'Italien et l'Anglais. Le Tasse et Milton lui étaient aussi familiers que Virgile : elle fit moins de progrès dans l'Espagnol, parce qu'on lui dit qu'il n'y a guères, dans cette Langue, qu'un livre célèbre, et que ce Livre est frivole.

L'étude de sa Langue fut une de ses principales occupations : il y a d'elle des remarques manuscrites, dans lesquelles on découvre, au milieu de l'incertitude de la grammaire, cet esprit philosophique qui doit dominer partout, et qui est le fil de tous les labyrinthes.

Parmi tant de travaux que le savant le plus laborieux eût à peine entrepris, qui croirait qu'elle trouvât du temps, non seulement pour remplir tous les devoirs de la société, mais pour en rechercher avec avidité tous les amusements ? Elle se livrait au plus grand monde comme à l'étude : tout ce qui occupe la société était de son ressort, hors la médisance. Jamais on ne l'entendit relever un ridicule, elle n’avait ni le temps, ni la volonté de s'en apercevoir ; et quand on lui disait que quelques personnes ne lui avaient pas rendu justice, elle ré- 
pondait qu'elle voulait l'ignorer. On lui montra un jour je ne sais quelle misérable brochure dans laquelle un auteur, qui n'était pas à portée de la connaître, avait osé mal parler d'elle. Elle dit que si l'auteur avait perdu son temps à écrire ces inutilités, elle ne voulait pas perdre le sien à les lire, et le lendemain ayant su qu'on avait renfermé l'auteur de ce libelle, elle écrivit en sa faveur, sans qu'il l'ait jamais su.

Elle fut regrettée à la Cour de France, autant qu'on peut l'être dans un pays où les intérêts personnels font si aisément oublier tout le reste. Sa mémoire a été précieuse à tous ceux qui l'ont connue particulièrement, et qui ont été à portée de voir l'étendue de son esprit et la grandeur de son âme.

Il eût été heureux pour ses amis qu'elle n'eût pas entrepris cet ouvrage dont les savants vont jouir. On peut dire d'elle, en déplorant sa destinée, periit arte sua.

Elle se crut frappée à mort longtemps avant le coup qui nous l'a enlevée : dès lors elle ne songea plus qu'à employer le peu de temps qu'elle prévoyait lui rester à finir ce qu'elle avait entrepris, et à dérober à la mort ce qu'elle regardait comme la plus belle partie d'ellemême. L'ardeur et l'opiniâtreté du travail, des veilles continuelles, dans un temps où le repos l'aurait sauvée, amenèrent enfin cette mort qu'elle avait prévue. Elle sentit sa fin approcher, et par un mélange singulier de sentiments qui semblaient se combattre, on la vit regretter la vie, et regarder la mort avec intrépidité : la douleur d'une séparation éternelle affligeait sensiblement son âme, et la Philosophie dont cette âme était remplie lui laissait tout son courage. Un homme qui s'arrachant tristement à sa famille qui le pleure, et qui fait tranquillement les préparatifs d'un long voyage, n'est que le faible portrait de sa douleur et de sa fermeté : de sorte que ceux qui furent les témoins de ses derniers moments sentaient doublement sa perte par leur propre affliction et par ses regrets, et admiraient en même temps la force de son esprit, qui mêlait à des regrets si touchants une confiance si inébranlable. 
Table des matières

\section{PRÉfACE DE MONSIEUR NEWTON}

\section{à la première édition des Principes en 1686}

Les anciens, comme nous l'apprennent Pappus ${ }^{1}$, firent beaucoup de cas de la mécanique dans l'interprétation de la nature, et les modernes ont enfin, depuis quelque temps, rejeté les formes substantielles et les qualités occultes, pour rappeler les Phénomènes naturels à des lois mathématiques. On s'est proposé dans ce Traité de contribuer à cet objet, en cultivant les Mathématiques en ce qu'elles ont de rapport avec la Philosophie naturelle.

Les anciens partagèrent la Mécanique en deux classes ; l'une théorique, qui procède par des démonstrations exactes ; l'autre pratique. De cette dernière ressortent tous les arts qu'on nomme Mécaniques, dont cette science a tiré sa dénomination : mais comme les Artisans ont coutume d'opérer peu exactement, de là est venu qu'on a tellement distingué la Mécanique de la Géométrie, que tout ce qui est exact, s'est rapportée à celle-ci, et ce qui l'était moins, à la première. Cependant les erreurs que commet celui qui exerce un art, sont de l'artiste et non de l'art. Celui qui opère moins exactement est un Mécanicien moins parfait, et conséquemment celui qui opérera parfaitement, sera le meilleur.

La Géométrie appartient en quelque chose à la Mécanique ; car c'est de cette dernière que dépend la description des lignes droites et des cercles sur lesquels elle est fondée. Il est effectivement nécessaire que celui qui veut s’instruire dans la Géométrie sache décrire ces lignes avant de prendre les premières leçons de cette science : après quoi on lui apprend comment les problèmes se résolvent par le moyen de ces opérations. On emprunte de la Mécanique leur solution : la Géométrie enseigne leur usage, et se glorifie du magnifique édifice qu'elle élève en empruntant si peu d'ailleurs. La Géométrie est donc 
fondée sur une pratique mécanique, et elle n'est autre chose qu'une branche de la Mécanique universelle qui traite et qui démontre l'art de mesurer. Mais comme les Arts usuels s'occupent principalement à remuer les corps, de là il est arrivé que l'on a assigné à la Géométrie, la grandeur pour objet, et à la Mécanique, le mouvement: ainsi la Mécanique théorique sera la science démonstrative des mouvements qui résultent des forces quelconques, des forces nécessaires pour engendrer des mouvements quelconques.

Les anciens qui ne considérèrent guères autrement la pesanteur que dans le poids à remuer, cultivèrent cette partie de la Mécanique dans leurs cinq puissances qui regardent les arts manuels; mais nous qui avons pour objet, non les Arts, mais l'avancement de la Philosophie, ne nous bornant pas à considérer seulement les puissances manuelles, mais celles que la nature emploie dans ses opérations, nous traitons principalement de la pesanteur, la légèreté, la force électrique, la résistance des fluides et les autres forces de cette espèce, soit attractives, soit répulsives : c'est pourquoi nous proposons ce que nous donnons ici comme les principes Mathématiques de la Philosophie naturelle. En effet toute la difficulté de la Philosophie paraît consister à trouver les forces qu'emploie la nature, par les Phénomènes du mouvement que nous connaissons, et à démontrer ensuite, par là, les autres Phénomènes. C'est l'objet qu'on a eu en vue dans les propositions générales du $\mathrm{I}^{\mathrm{er}}$ et $\mathrm{II}^{\mathrm{e}}$ livre, et on en donne un exemple dans le $\mathrm{III}^{\mathrm{e}}$ en expliquant le système de l'Univers : car on y détermine par les propositions Mathématiques démontrées dans les deux premiers livres, les forces avec lesquelles les corps tendent vers le Soleil et les Planètes; après quoi, à l'aide des mêmes propositions Mathématiques, on déduit de ces forces, les mouvements des Planètes, des Comètes, de la Lune et de la Mer. Il serait à désirer que les autres Phénomènes que nous présente la nature, pussent se dériver aussi heureusement des principes mécaniques : car plusieurs raisons me portent à soupçonner qu'ils dépendent tous de quelques forces dont les causes sont inconnues, et par lesquelles les particules des corps sont poussées les unes vers les autres, et s'unissent en figures régulières ou sont repoussées et se fuient mutuellement ; et c'est l'ignorance où l'on a été jusqu'ici de ces forces, qui a empêché les Philosophes de tenter l'explication de la nature avec succès. J'espère que les principes que j'ai posés dans cet Ouvra- 
ge pourront être de quelque utilité à cette manière de philosopher, ou à quelque autre plus véritable, si je n’ai pas touché au but.

L’ingénieux M. Halley, dont le savoir s'étend à tous les genres de littérature, a non seulement donné ses soins à cette Édition, en corrigeant les fautes de l'impression, et en faisant graver les figures : mais il est celui qui m’a engagé à la donner. Car après avoir obtenu de moi ce que j'avais démontré sur la forme des orbites planétaires, il ne cessa de me prier d'en faire part à la Société Royale, dont les instances et les exhortations gracieuses me déterminèrent à songer à publier quelque chose sur ce sujet. J'y travaillai ; mais après avoir entamé la question des irrégularités de la Lune, et diverses autres concernant les lois et la mesure de la pesanteur et des autres forces, les figures que décriraient les corps attirés par des forces quelconques, les mouvements de plusieurs corps entre eux, ceux qui se font dans des milieux résistants, les forces, les densités et les mouvements de ces milieux, les orbes enfin des Comètes; je pensai qu'il était à propos d'en différer l'édition jusqu'à un autre temps, afin d'avoir le loisir de méditer sur ce qu'il restait à trouver, et de donner un ouvrage complet au public : ce que je fais à présent. À l'égard des mouvements lunaires, ce que j'en dis étant encore imparfait, je l'ai renfermé dans les corollaires de la proposition LXVI du I ${ }^{\mathrm{er}}$ Livre, de crainte d'être obligé d'exposer et de démontrer chaque point en particulier : ce qui m'aurait engagé dans une prolixité superflue, et aurait troublé la suite des propositions.

J'ai mieux aimé placer dans quelques endroits, quoique peu convenables, des choses que j'ai trouvées trop tard, plutôt que de changer le numéro des propositions et des citations qui s’y rapportaient.

Je prie les savants de lire cet Ouvrage avec indulgence, et de regarder les défauts qu'ils y trouveront, moins comme dignes de blâme, que comme des objets qui méritent une recherche plus approfondie et de nouveaux efforts.

À Cambridge, du Collège de la Trinité, le 8 Mai 1686

ISAAC

NEWTON 


\section{Préface de l'auteur \\ à la tête de la seconde Édition}

Cette seconde Édition paraît corrigée dans plusieurs Articles et avec quelques additions. Dans la seconde Section du premier Livre on a rendu plus facile la manière de trouver les forces nécessaires pour faire mouvoir un corps dans des orbites données ; et dans la Section $\mathrm{VII}^{\mathrm{e}}$ du second Livre, on a recherché avec plus de soin la théorie de la résistance des fluides, qu'on confirme par de nouvelles expériences. Dans le III $^{\mathrm{e}}$ Livre, on déduit d'une façon plus complète la théorie de la Lune et la précession des Équinoxes, et l'on a ajouté à la théorie des Comètes un plus grand nombre d'exemples d'orbites calculées, et avec plus de soin : ce qui lui donne une nouvelle confirmation.

À Londres, le 28 Mars

1713

ISAAC

NEWTON 


\section{Préface de l'auteur à la troisième édition}

Dans cette troisième Édition, dont a eu soin M. Camberton, Docteur en médecine, très habile dans ces matières; on explique plus au long quelques points concernant la résistance des milieux, et on a ajouté quelques nouvelles expériences sur la chute des graves dans l'air. On explique aussi avec plus de détail dans le Livre troisième la démonstration qui prouve que la Lune est retenue dans son orbite par la force de gravité. Le même Livre est augmenté des observations nouvelles faites par $\mathrm{M}$. Pound sur la proportion des axes de Jupiter entre eux, de même que de quelques autres concernant la Comète de 1680, faites en Allemagne par M. Kirch, et qui ne nous sont parvenues que depuis peu. Elles montrent de nouveau combien les orbites paraboliques approchent de celles des Comètes. On détermine avec plus d'exactitude l'orbite de cette comète fameuse, suivant les calculs de M. Halley, et cela dans l'ellipse ; d'où l'on fait voir que cette comète se mouvant dans une orbite de cette forme, eut pendant neuf signes, un cours qui ne fut pas moins régulier que celui des planètes dans leurs orbites propres. On y a enfin ajouté la détermination de l'orbite de la Comète de 1723, calculée par $\mathrm{M}$. Bradley, Professeur d'Astronomie à Oxford.

À Londres, le 12 janvier 1725-6

ISAAC

NEWTON 
Table des matières

\section{PrÉfACE DE M. Cotes}

\section{Sur la présente Édition des Principes mathématiques de la Philosophie Naturelle de M. NEWTON.}

Nous donnons enfin au Public une nouvelle Édition de la Philosophie de M. de Newton, désirée depuis longtemps, et supérieure aux précédentes, par les corrections et les augmentations que l'Auteur y a faites. La Table des Matières est suffisante pour faire connaître au Lecteur tout ce que renferme cet excellent Ouvrage ; et la Préface de M. Newton l'instruira pareillement des additions et des changements qu'il a jugé nécessaires et convenables. Nous n'avons donc ici qu’à exposer en peu de mots quelle est la méthode dont il fait usage dans cette nouvelle Philosophie.

On peut rapporter à trois différentes classes tous les Auteurs qui ont entrepris de traiter la Physique. On a vu d'abord, des Philosophes qui ont donné à chaque espèce particulière de corps des qualités occultes et propres à chacun, d'où ils ont ensuite fait dépendre d'une manière encore plus occulte les Phénomènes dont nous sommes témoins. C'est là le fondement de la Philosophie de l'École, enseignée par Aristote et par les Péripatéticiens. Selon eux, chaque effet particulier dépend absolument d'une certaine Nature propre à chacun des corps qui en est le sujet ou la cause ; mais ils gardent un profond silence sur la cause et le principe de cette Nature. Puis donc qu'ils ont laissé les choses pour ne s'occuper que des mots ; on ne doit les regarder tout au plus que comme les inventeurs d'une espèce de jargon philosophique, et non comme les auteurs d'une véritable Philosophie.

D'autres ont pris le parti d'abandonner des mots vides de sens, et se sont flattés d'acquérir une gloire plus solide par des travaux plus réels. Ils ont donc posé pour principe, que toute la matière en général est de même nature ou homogène ; et que la variété que l'on remarque dans tout corps en particulier par sa configuration extérieure, ne dépend que de quelques affections très simples en elles-mêmes, et très 
faciles à concevoir. Rien de mieux que de procéder ainsi du plus simple au plus composé ; pourvu néanmoins que l'on ne donne pas à ces propriétés primitives et primordiales d'autres modes ni d'autres bornes que celles que la Nature a prescrites elle-même. Mais bientôt ces derniers Philosophes admirent à leur gré telles grandeurs et telles figures qu'ils jugèrent à-propos ; imaginèrent au besoin des mouvements et des positions respectives dans les parties composantes des corps: enfin ils forgèrent des fluides invisibles, doués d'une subtilité miraculeuse, agités par des mouvements secrets, capables de pénétrer les pores de tous les Corps, comme si la matière n’opposait aucune résistance ; et par là ils tombèrent dans des rêveries aussi ridicules que celles des Anciens, en négligeant de s'instruire et d'examiner la véritable constitution de la nature ; connaissance qu'on ne doit pas assurément chercher dans des conjectures trompeuses, puisque les observations les plus incontestables ont encore bien de la peine à nous la procurer.

Venons à la troisième classe, à ceux qui dans leur Philosophie ne reconnaissent d'autre règle que l'expérience. Ces derniers, bien convaincus que l'on doit, autant qu'il est possible, faire dépendre les effets des causes les plus simples, n'admettent cependant aucun principe qui ne soit prouvé par des observations constantes. Ils ne font point d'hypothèses, et n'en reçoivent aucunes en physique, si ce n'est pour les soumettre à l'examen et reconnaître leur vérité ou leur fausseté par une discussion exacte et rigoureuse. Ils emploient dans cette recherche les deux méthodes connues de tout le monde, l'Analyse et la Synthèse. Avec le secours de la première, de quelques Phénomènes choisis adroitement, ils déduisent les forces de la Nature, et les lois les plus simples qui dérivent de ces mêmes forces; ils exposent ensuite synthétiquement l'ordre et la disposition des autres qui dépendent immédiatement de ces premières. C'est là sans doute la meilleure Philosophie, et c'est aussi celle qu'a choisie notre illustre Auteur et qu'il a cru justement préférable à toute autre. C'est la seule qu'il ait jugée digne de ses soins et de ses travaux, et qu'il ait cru devoir perfectionner et embellir. L'explication du système du Monde qui se déduit si facilement de sa Théorie de la gravité, est à la fois une heureuse application de cette nouvelle philosophie, et un modèle que l'on ne peut trop imiter. Quelques Philosophes, avant M. Newton, ont soupçonné que la pesanteur pouvait être une propriété commune à tous les corps ; d'autres l'ont imaginé gratuitement : notre Philosophe est le premier 
et le seul qui ait pu le démontrer par les Phénomènes, et en faire le fondement inébranlable des Théories les plus brillantes.

Je n'ignore pas que des personnages illustres et de grand nom dans les Sciences n’ont accordé qu'avec peine leur suffrage à ce nouveau principe ; peut-être par un effet de certains préjugés, qui faisaient une impression trop forte sur leur esprit : je sais même, qu'ils ont quelquefois préféré des conjectures vagues à des vérités certaines. Mon dessein n'est point d'attaquer ici leur réputation, mais seulement de mettre mon Lecteur en état de porter un jugement équitable, par une exposition abrégée des découvertes du Chevalier Newton, sur la matière dont il est question.

Commençons donc d'abord par ce qu'il y a de plus simple et de plus à notre portée : jetons les yeux sur notre globe, et voyons quelle est la nature de la gravité dans les Corps sublunaires ; afin d'être plus assurés dans nos recherches, lorsque nous en serons aux Corps célestes qui se trouvent si éloignés de notre habitation. Tous les Philosophes sont d'accord pour admettre une gravitation générale de tous les Corps terrestres vers notre globe. On est convaincu par un grand nombre d'expériences, qu'il n’y a pas de Corps vraiment léger. Ce que l'on appelle légèreté n'est qu'une propriété relative et apparente; ce n'est pas une légèreté absolue et véritable; on sait qu'elle dépend d'une gravité plus puissante des Corps environnants.

Cela posé, puisque les Corps gravitent vers la Terre, il saut aussi que la Terre gravite également vers les Corps ; car il est aisé de prouver , comme on va le faire tout à l'heure, que l'action de la gravité est égale et réciproque. Imaginons la masse de la Terre partagée en deux parties quelconques, égales ou inégales. Si les efforts ou les poids de chaque partie l'une vers l'autre n'étaient pas égaux, la plus faible cèderait nécessairement à la plus forte, et les deux parties ainsi unies continueraient de se mouvoir à l'infini vers le point du ciel opposé à la direction de la plus pesante; ce qui est absolument contraire à l'expérience ; il faut donc dire que les poids des parties sont dans un parfait équilibre, c'est-à-dire, que l'action de la gravité est égale et réciproque.

Les poids des corps également éloignés du centre de la Terre sont comme les quantités de matière qu'ils renferment. C'est une suite né- 
cessaire de l'égalité d'accélération des corps qui tombent par la seule force de leur pesanteur ; car il est évident que des forces qui impriment à des corps inégaux des degrés égaux de vitesse, doivent être proportionnelles à la quantité de matière qu'il faut mettre en mouvement. D'ailleurs on est maintenant assuré que tous les corps reçoivent une égale accélération ; puisque, dans le vide de Boile, ils décrivent tous des espaces égaux en temps égaux; n'étant plus différemment arrêtés par la résistance de l'air. La même vérité est encore prouvée avec plus d'exactitude par l'expérience des pendules.

Les forces attractives ${ }^{2}$ des corps à distances égales sont comme les quantités de matière contenues dans ces mêmes corps. Car puisque les corps gravitent vers la Terre, et que celle-ci gravite vers les corps avec des moments égaux, le poids de la Terre sur un corps quelconque, ou, ce qui est la même chose, la force avec laquelle un corps attire la Terre, sera égale à la pesanteur de ce même corps vers la Terre. Mais dans chaque corps, le poids est proportionnel à la quantité de matière : donc la force avec laquelle un corps attire la Terre, ou, ce qui revient au même, la force absolue de ce corps sera comme la même quantité de matière qu'il renferme.

Il suit de là que la force attractive (ou la pesanteur) des corps résulte des forces attractives (ou des pesanteurs ) de chaque partie qui les composent ; puisque cette force de gravitation augmente ou diminue selon que la quantité de matière augmente ou diminue. Il faut regarder l'action de la Terre comme le résultat des actions réunies de toutes ses parties ; et par conséquent il faut que tous les corps terrestres s'attirent avec des forces absolues qui soient en raison de la matière attirante.

On remarquera ici que $\mathrm{M}$. Côtes emploie le mot de force attractive pour exprimer la pesanteur, comme a fait M. Newton. En général, toutes ces expressions, force attractive, attraction, gravité, gravitation, pesanteur, ne signifient rien autre chose que cette tendance de tous les corps vers un centre commun de pesanteur, soit que cette tendance qui produit réellement une force, soit occasionnée dans les corps par un mécanisme que nous ignorons ; soit que plutôt elle soit une propriété continuellement imprimée à la matière par un pur effet de la volonté du Créateur, qui veut produire par là tous les Phénomènes dont nous sommes témoins. Il ne s’agit ici que du fait ; les noms sont indifférents et présenteraient tous les mêmes difficultés pour quiconque n’entrerait pas bien dans l'esprit de l'Auteur. Voyez à ce sujet le Chapitre II. des Discours de M. de Maupertuis, sur la Fig. des Astres, pag. 16 de la nouvelle édition. On ne peut rien de plus lumineux que cet excellent morceau, qui est une discussion (vraiment) métaphysique sur l’attraction, comme son titre l'annonce. 
Telle est la nature de la gravité sur la Terre : voyons maintenant ce qu'elle est dans les cieux.

C'est une loi de la Nature reçue de tous les Philosophes, qu'un corps restera toujours en repos, ou continuera de se mouvoir en ligne droite, tant qu'il ne sera point soumis à l'action de forces étrangères qui l'obligent de changer de situation. Il suit de là que les corps qui se meuvent dans des courbes, et qui par conséquent s'écartent continuellement des lignes droites qui touchent leurs orbites, sont aussi continuellement retenus dans cette route curviligne par l'action d'une force qui leur est perpétuellement appliquée. Donc, pendant que les planètes décrivent leurs trajectoires, elles seront continuellement détournées des tangentes à chaque point de la courbe, par l'action répétée d'une force toujours présente.

Il y a encore un principe qu'il faut accorder, et que l'on démontre géométriquement, c'est que lorsque des corps mus dans une courbe qui se trouve sur un même plan décrivent autour d'un point fixe ou mobile des aires proportionnelles aux temps, ils sont poussés par des forces qui tendent vers ce même point : donc puisque tous les Astronomes conviennent que les Planètes principales décrivent autour du soleil des aires proportionnelles aux temps, de même que les satellites de chacune de ces Planètes du premier ordre autour de ces mêmes Planètes ; il faut conclure que la force qui les détourne continuellement des tangentes de leurs orbites pour les faire circuler dans ces mêmes courbes, est aussi continuellement dirigée vers les corps qui se trouvent aux foyers de ces orbites. C'est donc avec raison que l'on peut appeler cette force une force centripète à l'égard du corps circulant ; et une force attractive à l'égard du corps central, quelle que soit la cause qui produit cette force.

De plus, il est pareillement démontré géométriquement que si plusieurs corps se meuvent uniformément dans des cercles concentriques, de manière que les carrés des temps périodiques soient entre eux comme les cubes des distances au centre commun ; les forces de chacun de ces corps seront réciproquement comme les carrés des mêmes distances. On démontre avec la même facilité que, si des corps font leurs révolutions dans des orbites qui ne diffèrent presque pas du cercle, et dont les absides soient fixes ; les forces centripètes de ces corps seront comme les carrés des distances. Or de l'aveu constant de tous 
les Astronomes toutes les planètes se trouvent dans l'un ou l'autre cas ; donc leurs forces centripètes sont réciproquement comme les carrés de leurs distances au centre. Si l'on nous objecte que les absides des orbites de chaque Planète, et particulièrement de la Lune, ne sont pas dans un repos parfait ; mais qu'ils ont un mouvement fort lent suivant l'ordre des signes ; on peut répondre que, quand même nous accorderions que cette erreur vient de ce que la loi de la force centripète s'éloigne tant soit peu de la raison doublée inverse des distances; néanmoins il est aisé de calculer jusqu'où peut aller l'erreur qui suit de cette fausse supposition, et de faire voir qu'elle est absolument insensible. En effet, quoique la loi de la force centripète de la Lune qui est la plus sujette à être troublée dans ses mouvements, surpasse un peu le rapport de la raison doublée; néanmoins elle en approche soixante fois davantage que de la raison triplée. On peut encore réfuter cette objection plus solidement en soutenant, comme il est démontré dans cet Ouvrage, que ce mouvement des absides ne vient pas de ce que l'intensité des forces centripètes s’éloigne de la raison doublée, mais qu'il dépend réellement d'une cause totalement différente : ainsi il faudra toujours admettre comme un principe incontestable, que les Planètes principales tournent autour du Soleil, et les secondaires autour des premières, par l'action de forces centripètes qui suivent précisément la raison inverse des carrés des distances.

De ce que l'on vient de dire, il suit évidemment que les Planètes sont retenues dans leurs orbites par une force qui agit continuellement sur elles ; que cette force est toujours dirigée vers le centre de ces orbites ; qu'elle augmente à mesure que les Planètes approchent du centre, et qu'elle diminue à mesure qu'elles s'en éloignent; que l'augmentation croît comme le carré de la distance décroît. Examinons présentement par une comparaison bien établie, si la pesanteur qui fait tomber les corps sur notre globe n'est pas de même nature que les forces centripètes qui retiennent les Planètes dans leurs orbites. Le moyen de s'en assurer, c'est de voir si l'on ne pourra pas trouver de part et d'autre les mêmes lois et les mêmes propriétés : pour y parvenir, commençons par chercher quelle est la force centripète de la lune qui est le corps le plus proche de notre globe.

Les espaces rectilignes parcourus en tombant par des corps quelconques depuis le point de repos, pendant un temps donné, sont proportionnels aux forces qui les poussent ; c'est une proposition démon- 
trée dans toute la rigueur géométrique : donc la force centripète de la Lune parcourant son orbite, sera à la force de la pesanteur sur la surface de la Terre, comme l'espace que la lune décrirait en descendant vers la Terre, dans un temps infiniment petit en vertu de sa force centripète, si elle n'avait point de mouvement de révolution, est à l'espace que parcourt dans le même temps un corps près de la surface de la Terre par la seule force de la pesanteur. Le premier des espaces dont on vient de parler est égal au sinus-verse de l'arc décrit par la lune, pendant le même temps ; puisque ce sinus-verse mesure la quantité dont la force centripète a écarté la lune de la tangente ; cet espace peut se calculer par la connaissance du temps périodique de la lune et de sa distance au centre. L'autre espace dont nous avons parlé, se déduit de la théorie des pendules, suivant les expériences de M. Huygens. Si l'on sait le calcul on trouvera que le premier espace est au second, ou, ce qui revient au même, que la force centripète qui retient la lune dans son orbite est à la force de la pesanteur sur la surface de la Terre, comme le carré du demi-diamètre de la Terre est au carré du demi-diamètre de l'orbite de la lune. D'ailleurs, suivant ce qui précède, la force centripète de la lune dans son orbite est à la force centripète de la lune auprès de la surface de la Terre dans la même raison ; donc la force centripète de la lune et la force de la pesanteur sur la surface de la Terre sont entièrement égales. Ce ne sont donc point deux forces distinctes et différentes, mais précisément une seule et même force ; car si ces deux forces avaient lieu en même temps et se trouvaient néanmoins distinguées l'une de l'autre près de la surface de la Terre, les corps tomberaient deux fois plus vite que par la seule force de la pesanteur. Il est donc certain que cette force centripète qui retient la lune dans son orbite en l'écartant de la tangente, par attraction ou par impulsion, n'est autre chose que la force de la pesanteur terrestre qui s'étend jusques à la lune ; et la raison seule nous fait voir que cette force peut avoir son effet à des distances encore plus grandes, puisque nous ne pouvons pas observer la moindre diminution sensible au sommet des plus hautes montagnes. La lune gravite donc vers la Terre, et par une action réciproque la Terre gravite vers la lune : on verra cette proposition confirmée dans cet Ouvrage, lorsqu'il est question du flux et reflux de la mer et de la précession des Équinoxes, Phénomènes qui dépendent tous deux de l'action combinée de la lune et du soleil sur la Terre. Cette même comparaison que l'on vient de faire nous apprend en même temps la loi suivant laquelle dé- 
croît la force de la pesanteur dans les grandes distances de la Terre ; car puisque la pesanteur des corps terrestres ne diffère pas de la force centripète de la lune, qui décroît en raison des carrés des distances ; la pesanteur suivra donc aussi la même loi, et diminuera dans la même proportion.

Venons présentement aux autres planètes. Puisque les révolutions des planètes principales autour du soleil, celles des Satellites de Jupiter et de Saturne, autour de ces deux Planètes sont des phénomènes de même nature que la révolution de la lune autour de la Terre ; puisqu'il est démontré de plus que les forces centripètes de ces planètes sont dirigées vers le centre du soleil, et que celles des Satellites de Jupiter et de Saturne sont pareillement dirigées vers le centre de chacune de ces deux planètes, comme la force centripète de la lune est elle-même dirigée vers le centre de la Terre ; enfin puisque toutes ces forces sont réciproquement proportionnelles aux carrés de leurs distances, de même que la force de la lune (comparée à celle des corps terrestres) est réciproquement comme le carré de la distance : il faudra donc conclure que toutes ces forces sont de même espèce. Ainsi de même que la lune gravite sur la Terre, et la Terre sur la lune ; de même aussi toutes les planètes secondaires graviteront vers les planètes principales, et celles-ci graviteront toutes vers leurs Satellites ; de même enfin toutes les planètes graviteront vers le soleil, et le soleil gravitera vers toutes les planètes.

Il faut donc reconnaître que le soleil gravite sur toutes les planètes, et que toutes les planètes pèsent réciproquement sur celui-ci. Car pendant que les Satellites accompagnent leur planète principale, ils font en même temps leurs révolutions autour du soleil, ainsi que cette même planète : donc il est prouvé par le même raisonnement que les planètes principales et secondaires pèsent vers le soleil, et que le soleil pèse vers elles. On a encore outre cela d'autres preuves de la pesanteur des planètes secondaires vers le soleil, déduites des inégalités du mouvement de la lune, dont on trouvera une théorie exacte exposée avec toute la sagacité possible dans la troisième partie de cet Ouvrage.

On peut encore déduire du mouvement des Comètes que la force attractive du soleil se sait sentir à des distances énormes dans toutes les parties de l'étendue. En effet ces corps, après avoir parcouru un intervalle immense, s'approchent continuellement du soleil ; et quel- 
quefois ils sont si près de ce globe qu'ils paraissent presque le toucher lorsqu'ils se trouvent dans leur périhélie. C'est en vain que les Astronomes des siècles précédents ont cherché à établir une théorie de ces nouvelles planètes ; cette découverte était réservée à notre siècle, et à notre illustre Auteur, qui nous a donné des méthodes aussi faciles dans la pratique qu'elles sont conformes aux observations. Il est donc évident que les Comètes se meuvent dans des sections coniques qui ont leur foyer au centre du soleil ; et que les rayons menés du soleil aux différents points de leurs trajectoires décrivent des aires proportionnelles aux temps. Il suit encore évidemment de ces Phénomènes, et l'on peut aussi le démontrer géométriquement, que les forces qui retiennent les Comètes dans leurs orbites sont dirigées vers le soleil, et que leur intensité est en raison inverse des carrés de leurs distances au centre de ce même astre. Donc les Comètes gravitent vers le soleil, et par conséquent la force attractive du soleil s'étend non seulement aux différentes planètes qui se trouvent à des distances finies, et qui sont presque toutes dans un même plan ; mais elle agit encore sur les Comètes qui se trouvent placées dans toutes les différentes parties du Ciel, et à toutes sortes de distances. Telle est donc la nature des corps pesants, qu'ils sont sentir leur action à toutes les distances imaginables sur tous les autres corps pesants. Il suit encore de là que les Planètes et les Comètes s'attirent mutuellement, et que tous ces corps gravitent réciproquement les uns vers les autres; et cette conséquence se trouve confirmée par les inégalités des mouvements de Jupiter et de Saturne, connues des Astronomes, et causées par les actions réciproques de ces planètes les unes sur les autres. Le mouvement si lent des apsides, et dont on a parlé ci-devant, vient encore à l'appui de cette vérité, et dépend de causes entièrement semblables.

Il faut reconnaître maintenant d'après tout ce que l'on vient de voir, que la Terre, le soleil et tous les corps célestes qui accompagnent le soleil ont une gravitation réciproque les uns vers les autres, par laquelle ils paraissent s'attirer. Donc chacune de leurs parties, si petite qu'elle soit, a pareillement une force d'attraction proportionnelle à sa masse, suivant ce que l'on a dit plus haut sur les corps terrestres : à différentes distances, les forces de ces mêmes parties seront réciproquement comme les carrés des distances ; car il est encore démontré que les globes qui attirent, suivant cette loi, doivent être composés de parties attirantes dans la même raison. 
Les conséquences que l'on vient de déduire, sont fondées sur cet axiome reçu de tous les Philosophes, que les effets de même genre dont les propriétés connues sont les mêmes, ont aussi les mêmes causes, d'où naissent les mêmes propriétés, quoique ces causes ne soient pas encore connues. Qui doute en effet, si c'est la pesanteur qui fait tomber les pierres en Europe, que ce ne soit aussi la même pesanteur qui les fasse tomber en Amérique ? Si la pesanteur est réciproque entre la Terre et les pierres en Europe, qui pourra nier qu'elle ait la même propriété en Amérique ? Si la force attractive de la Terre ou d'une pierre est le résultat des forces attractives des parties dans l'Europe ; ne faut-il pas aussi qu'en Amérique elle résulte d'une pareille combinaison ? Si la force de la pesanteur se trouve dans toutes les espèces de corps, et se fait sentir à toutes sortes de distances en Europe, pourquoi voudrions-nous soutenir qu'elle n'aurait pas aussi les mêmes propriétés en Amérique ? Cette règle est la base de toute la Philosophie ; supprimez-la et vous ne pourrez plus rien établir d'universel. On ne connaît la nature de chaque chose que par les observations et les expériences, et de là il suit que nous ne jugeons que par cette règle d'analogie.

Puis donc que tous les corps terrestres et célestes que nous pouvons observer, ou sur lesquels nous pouvons faire des expériences, sont des corps pesants ; il faudra dire que la pesanteur est une propriété qui convient à tous les corps ; et de même que nous n'en pouvons concevoir aucuns qui ne soient étendus, mobiles et impénétrables, nous ne pouvons pas non plus en concevoir qui ne soient pesants. C'est par l'expérience que nous connaissons l'étendue, la mobilité et l'impénétrabilité des corps, et c'est aussi par l'expérience que nous connaissons leur gravité. Tous les corps que nous avons pu observer sont étendus, mobiles et impénétrables; et nous en concluons que tous, ceux mêmes sur lesquels nous n'avons pas pu faire d'observations, sont pareillement étendus, mobiles et impénétrables. Tous les corps que nous avons pu observer sont pesants, et nous concluons légitimement de même que ceux sur lesquels nous n'avons point fait d'expériences, sont aussi des corps pesants. Si l'on nous dit que les corps des étoiles fixes n'ont point de gravité, parce que l'on n'a pas encore pu l'observer, on pourra nous prouver aussi par le même raisonnement que ces corps ne sont ni étendus, ni mobiles, ni impénétrables; car on n’a pas encore observé ces propriétés dans les 
fixes. Mais à quoi bon m’arrêter plus longtemps ? il faut que la pesanteur soit une des propriétés primitives de tous les corps, ou que l'on cesse de regarder comme telle leur étendue, leur mobilité, leur impénétrabilité ; il faut que l'on puisse expliquer exactement les phénomènes de la nature par la loi de la pesanteur, ou que l'on renonce à en donner une explication raisonnable en faisant usage de l'étendue, de la mobilité et de l’impénétrabilité des corps.

Je ne doute pas qu'on ne désapprouve cette conclusion, et qu'on ne me reproche de ramener les qualités occultes. On ne cesse de nous objecter que la gravité est une qualité de cette espèce, et qu'on doit bannir absolument de la philosophie toutes les explications fondées sur de pareilles causes : mais nous pouvons répondre que l'on ne doit pas appeler occultes des qualités dont l'existence est évidemment démontrée par l'expérience ; mais celles-là seulement qui n'en ont qu'une imaginaire, et qui ne sont prouvées en aucune manière. Ceux qui ont réellement recours aux qualités occultes sont ceux qui, pour expliquer les mouvements de la nature, ont imaginé des tourbillons d'une matière qu'ils forgent à plaisir, et qui ne tombe sous aucun sens.

Faudra-t-il donc rejeter la gravité de tous les ouvrages philosophiques, comme une qualité occulte, par ce que l'on ignore jusqu'à présent la cause de cette même gravité ? En établissant de pareils principes, que l'on prenne garde de donner dans des absurdités manifestes, et de ruiner par là tous les fondements de la Philosophie. En effet toutes les causes sont liées les unes aux autres par une chaîne non interrompue, et se déduisent les unes des autres en allant du plus simple au plus composé. Si vous arrivez une fois à la cause la plus simple, il ne vous sera pas possible de remonter plus haut ; car on ne peut pas donner une explication mécanique de la cause la plus simple ; et si cela se pouvait, dès lors elle cesserait d'être telle. Il faudra donc traiter de qualités occultes les causes de cette nature, et les bannir de la Philosophie ; ce qui ne peut avoir lieu, que l'on n'exclue pareillement toutes celles qui dépendent immédiatement des premières, et celles qui se déduisent des secondes, et ainsi de suite jusqu'à ce que l'on ait absolument supprimé toutes les causes des phénomènes qu'il faut expliquer.

D’autres regardent la gravité comme un effet surnaturel, et veulent que ce soit un miracle perpétuel, d'où ils concluent qu'il faut la reje- 
ter, puisque les causes surnaturelles ne doivent point avoir lieu en physique. Une objection si misérable, et qui renverse toute philosophie, mérite à peine que l'on y réponde ; car suivant cette idée, ils se trouvent réduits à l'une de ces deux extrémités, ou de soutenir, contre toute évidence, que la pesanteur n'est pas une propriété commune à tous les corps ; ou de regarder comme surnaturel tout ce qui ne dépend pas des autres propriétés des corps, ou d'une cause mécanique. Il est cependant constant qu'il y a dans les corps des propriétés primitives, et qui par cette raison ne peuvent dépendre d'autres propriétés : que l'on examine donc si ces propriétés ne sont pas surnaturelles, et par conséquent dans le cas d'être rejetées ; qu'on voie enfin ce que deviendrait la Philosophie avec de tels raisonnements.

Il a encore une autre espèce de Philosophes qui ne rejettent la Physique céleste de $\mathrm{M}$. Newton que parce qu'elle est opposée au système de Descartes, et ne paraît pas pouvoir s'accorder avec les principes de ce Philosophe. Nous ne pouvons pas les empêcher de suivre leur sentiment ; mais il faut qu'ils se conduisent de même à notre égard, et qu'ils ne refusent pas aux autres une liberté qu'ils veulent qu'on leur accorde. Qu'il nous soit donc permis d'embrasser la Philosophie de Newton, et de nous y attacher, parce qu'elle nous paraît plus véritable; qu'il nous soit permis de préférer des causes prouvées par les phénomènes à des causes fictives, et qui ne sont confirmées par aucune expérience. Une vraie Philosophie ne doit employer dans l'explication de la nature que des causes vraiment existantes; elle ne doit point chercher les lois par lesquelles le Tout-puissant aurait pu produire l'ordre admirable qui règne dans cet univers, s'il avait jugé àpropos de les employer ; mais seulement celles qu'il a réellement établies par un acte libre de sa volonté. En effet, nous pouvons croire raisonnablement qu'un même effet peut être produit par plusieurs causes différentes ; mais la vraie cause pour un Philosophe, est celle qui produit actuellement l'effet dont il est question : la bonne Philosophie n'en reconnaît point d'autres. Dans les pendules, le même mouvement de l'aiguille qui marque les heures peut dépendre également d'un poids suspendu, ou d'un ressort enfermé dans la machine. Si l'on a devant soi une horloge mise en mouvement par un poids, ce serait une chose ridicule d'imaginer un ressort, et de vouloir expliquer le mouvement de l'aiguille par cette hypothèse faite avec trop de précipitation ; Car il fallait d'abord considérer attentivement la construction 
intérieure de la machine, afin de reconnaître par expérience le vrai principe du mouvement proposé : on peut porter à-peu-près le même jugement de ces Philosophes qui commencent par établir que l'espace immense des Cieux est rempli d'une matière extrêmement subtile, et veulent ensuite que cette même matière soit mise dans un mouvement continuel par les tourbillons qu'elle a formés ; car il pourrait arriver qu'ils expliquassent tous les Phénomènes par leurs hypothèses, et l'on ne pourrait pas dire pour cela qu'ils nous eussent donné une vraie Philosophie, ni qu'ils eussent découvert les vraies causes des mouvements célestes ; à moins qu'ils ne nous aient démontré l'une de ces deux propositions, ou que les causes qu'ils nous donnent existent réellement, ou qu'il n'en pourrait exister d'autres.

Si donc nous faisons voir que l'attraction des corps a réellement lieu dans la nature ; si nous montrons de plus comment on peut expliquer tous les mouvements célestes par cette propriété ; dès lors c'est nous faire une objection ridicule et sans force, que de vouloir nous prouver que l'on doit expliquer ces mêmes mouvements par les tourbillons, quand même nous aurions accordé la possibilité d'une telle explication. Mais il s'en faut de beaucoup, car on ne peut expliquer ces phénomènes en aucune manière par le moyen des tourbillons, c’est une chose si bien prouvée par notre Auteur, démontrée par des raisons si solides, que ce serait vouloir s'occuper sérieusement de rêveries que de consacrer sans aucun fruit son temps et ses travaux, à rétablir un édifice misérable et chimérique par des éclaircissements ou des commentaires également inutiles,

En effet, si les corps des Planètes et des Comètes sont emportés autour du Soleil par des tourbillons ; il faut que les corps emportés et les parties du tourbillon voisines de ces corps aient la même vitesse et la même direction ; il faut par conséquent qu'elles aient la même densité ou une même force directement proportionnelle à la quantité de matière. Or il est constant que les Planètes et les Comètes, lorsqu'elles se trouvent dans la même partie du Ciel, ont néanmoins des vitesses et des directions différentes. Il est donc nécessaire que les mêmes parties du fluide céleste, qui sont à égales distances du soleil tournent dans le même temps avec des directions et des vitesses différentes ; car il faut une direction et une vitesse déterminée pour le passage des Planètes ; et il faut dans le même temps une autre vitesse et une autre direction pour le passage des Comètes. Comme ce Phénomène est absolument 
inexplicable, de deux choses l'une, ou il faudra convenir que tous les corps célestes ne sont pas emportés par un tourbillon ; ou il faudra dire que ce n'est pas un seul tourbillon qui produit tous ces mouvements ; mais plusieurs qui sont différents les uns des autres, et qui occupent le même espace du Ciel, qu'ils parcourent dans le même temps avec des vitesses et des directions différentes.

Si l'on suppose qu'un même espace contient différents tourbillons, qui se pénètrent mutuellement et font leurs révolutions avec des mouvements différents; comme d'ailleurs tous les mouvements doivent être parfaitement analogues à ceux des corps qu'ils entraînent, lesquels font leurs révolutions avec une régularité surprenante dans des sections coniques tantôt fort excentriques, tantôt presque circulaires ; on peut demander avec raison comment il peut se faire que ces mouvements se conservent en entier sans jamais avoir été troublés depuis tant de siècles par les actions diverses de la matière qu'ils rencontrent sans cesse. Si de plus on fait attention que ces mouvements imaginaires sont plus composés et plus difficiles à expliquer que les mouvements réels et véritables des Planètes et des Comètes ; on sera bientôt convaincu, ainsi que nous, qu'ils ont été gratuitement introduits dans la Philosophie ; car toute cause doit être plus simple que son effet. Si l'on accorde une fois la liberté d'imaginer tout ce que l'on voudra, on verra bientôt quelqu'un nous assurer que toutes les Planètes et les Comètes sont ainsi que notre Terre environnées d'atmosphères; et d'abord cette hypothèse paraît plus conforme à la raison. On nous dira ensuite que ces atmosphères, par leur nature, se meuvent autour du soleil et décrivent des sections coniques ; et ce mouvement peut encore se concevoir plus facilement qu'un semblable mouvement propre à divers tourbillons qui se pénètrent mutuellement : enfin on établira bientôt, comme une chose absolument hors de doute, que les Planètes et les Comètes sont emportées autour du soleil par leurs atmosphères, et l'on triomphera d'avoir ainsi découvert les causes des mouvements célestes. Mais quiconque rejette une pareille fiction doit aussi à plus sorte raison rejeter la première ; car ces deux hypothèses n'en sont absolument qu'une seule.

Galilée a démontré qu'une pierre jetée et mue dans une parabole ne quitte la ligne droite que par la force de la pesanteur, qui est pourtant une qualité occulte. Mais il faut espérer que quelque Philosophe plus fin et plus adroit imaginera un jour une autre cause ; il supposera 
quelque matière subtile, invisible, impalpable, qui ne peut tomber sous aucun sens, mais qui se trouve dans les environs de la surface de la Terre ; il soutiendra que cette matière se meut dans toutes sortes de directions, qu'elle obéit à toutes sortes de mouvements différents et même opposés, et enfin qu'elle décrit toutes sortes de lignes paraboliques ; ensuite il aura bientôt expliqué d'une manière brillante pourquoi la pierre quitte la ligne droite ; et par là s'attirera l'approbation d'un vulgaire ignorant. Cette pierre, nous dira-t-il, nage dans un fluide subtil, et en suivant son cours, elle doit nécessairement se conformer au mouvement du milieu dans lequel elle se trouve. Or ce fluide se meut dans des lignes paraboliques; donc il faut absolument que la pierre décrive une parabole ? Qui n'admirera un si grand Philosophe, un génie si perçant ? est-il possible d'expliquer les Phénomènes de la nature d'une manière plus claire, plus à la portée même du commun, et enfin par des causes plus mécaniques, la matière et le mouvement ? Qui ne rira au contraire de ce pauvre Galilée, qui emploie le plus grand appareil de Géométrie pour ramener de nouveau des qualités occultes que l'on avait si sagement bannies de la Philosophie : mais rougissons de nous amuser à des puérilités de cette nature, et parlons enfin sérieusement.

Tout se réduit à ce qui suit : il y a un nombre infini de Comètes, leurs mouvements sont extrêmement réguliers, et elles suivent précisément les mêmes lois que les Planètes; elles se meuvent dans des sections coniques ; leurs trajectoires sont extrêmement excentriques ; il y en a dans toutes les parties du Ciel ; elles parcourent les espaces célestes, et passent auprès des Planètes avec la plus grande facilité ; souvent même elles marchent contre l'ordre des lignes : tous ces Phénomènes sont confirmés par les observations astronomiques, et ne peuvent s'expliquer par les tourbillons. Bien plus ils ne peuvent pas même exister si les Planètes se trouvent entraînées par des tourbillons ; enfin le mouvement des Comètes devient absolument impossible, si l'on ne bannit de l'univers, cette matière subtile qui ne doit son existence qu'à l'imagination, et si on ne la fait rentrer dans le néant dont on l'avait tirée.

Examinons encore cette matière et voyons plus en détail ce qui suit de l'hypothèse des tourbillons. Si les Planètes sont ainsi emportées autour du soleil ; suivant ce que l'on a déjà dit, les parties du tourbillon qui environnent la Planète doivent être de même densité qu'elle ; 
ainsi toute la matière qui environne le périmètre du grand orbe sera aussi dense que la Terre, et celle qui se trouve entre ce grand orbe et celui de Saturne aura autant ou plus de densité ; car pour qu'un tourbillon puisse subsister, il faut que les parties les moins denses soient vers le centre et que les plus denses s'en éloignent. En effet, puisque les carrés des temps périodiques des Planètes sont comme les cubes des distances au Soleil, il faut que les temps périodiques des parties de chaque tourbillon voisines de la Planète suivent à-peu-près le même rapport : or il suit de là que les forces centrifuges de ces mêmes parties sont en raison inverse des carrés des distances. Donc celles qui sont plus éloignées ont moins de force centrifuge, et par conséquent si elles ont moins de densité elles céderont à la plus grande force avec laquelle les parties plus voisines du centre tâchent de s'en écarter ; donc les plus denses monteront tandis que les moins denses descendront : il y aura ainsi un changement continuel de lieu jusqu'à ce que toute la matière du tourbillon se trouve tellement disposée qu'elle puisse demeurer en équilibre. Si deux fluides de différente pesanteur spécifique sont contenus dans un même vase, on sait que le plus pesant va toujours au fond ; et c'est par une raison presque toute semblable que les parties les plus denses d'un tourbillon s'écartent du centre en vertu d'une plus grande force centrifuge. Il faut donc reconnaître que toute la partie du tourbillon qui se trouve au-dehors de l'orbe de la Terre, par rapport au soleil, aura une densité et par conséquent une force d'inertie proportionnée à la quantité de matière, laquelle densité sera au moins égale à la densité et à l'inertie de notre Terre ; d'où il suit que les Comètes éprouveront une résistance considérable et très sensible dans leur mouvement, pour ne pas dire capable de le détruire absolument, comme cela est plus que probable. Il est néanmoins certain par la régularité des mouvements de ces mêmes Comètes, qu'elles n'éprouvent pas la moindre résistance sensible, et par conséquent qu'elles ne trouvent nulle part aucune matière qui puisse leur résister, ou ce qui revient au même, qui ait quelque densité ou quelque force d'inertie. Car la résistance des milieux ne vient que de l'inertie de la matière fluide, ou de la viscosité ou ténacité des parties de ce même fluide. Celle qui vient de cette dernière cause est très petite et peut à peine être observée dans les fluides connus, à moins que le degré de viscosité ou ténacité ne se trouve très considérable, comme cela se voit dans l'huile ou le miel. La résistance que l'on éprouve dans l'eau, dans l'air, dans le vif-argent et autres fluides de cette espè- 
ce qui n'ont point de viscosité est presque toute de même nature que celle dont nous avons parlé d'abord, et ne peut pas être diminuée par de nouveaux degrés de subtilité, tant que la densité à laquelle elle est toujours proportionnelle, reste la même. Tout ceci est démontré par notre illustre Auteur avec toute la clarté possible, dans sa belle Théorie de la résistance des milieux ; Théorie qui se trouve exposée avec beaucoup plus de précision dans cette nouvelle Édition, et qui est encore confirmée davantage par les expériences sur la chute des corps.

On sait que les corps en mouvement le communiquent peu à peu au fluide environnant ; cette communication produit une perte, et cette perte ralentît nécessairement la vitesse. La diminution de vitesse est donc proportionnelle au mouvement communiqué, lequel est luimême comme la densité du fluide lorsque la vitesse est connue : donc la diminution de mouvement ou la résistance sera aussi comme la même densité du fluide, et rien ne peut la supprimer, à moins que le fluide qui vient choquer les parties postérieures du corps en mouvement ne lui rende ce qu'il a perdu par la résistance du milieu. Mais c'est ce que l'on ne peut dire, à moins que l'impression du fluide sur les parties postérieures du corps ne soit égale à celle que le même corps exerce sur les parties du fluide qui lui sont directement opposées ; c'est-à-dire, à moins que la vitesse relative avec laquelle le fluide revient frapper le corps par derrière ne soit égale à celle avec laquelle le corps frappe le fluide ; ou, ce qui revient au même, à moins que la vitesse absolue du fluide récurrent ne soit double de celle du fluide repoussé par le corps ; ce qui est absolument impossible. On ne peut donc en aucune manière supprimer la résistance des fluides, du moins celle que produisent la densité et l'inertie; d'où il faut conclure que les fluides célestes n'ont aucune force d'inertie puisqu'ils n’opposent aucune résistance ; qu'il n'y a pareillement aucune force qui communique le mouvement, puisqu'il n'y a point de force d'inertie ; point de force qui puisse produire le plus léger changement dans les corps en général ou en particulier, puisqu'il n’y a point de force qui puisse communiquer le mouvement ; en un mot que ces fluides n'ont aucune efficacité, puisqu'ils n'ont aucun moyen de produire le changement. Pourquoi, donc ne pas regarder comme ridicule et indigne d'un Philosophe une hypothèse qui n'a point de fondement et ne peut en aucune manière servir à expliquer les lois et les phénomènes de la nature ? Ceux qui veulent que l'univers soit rempli de matière, et 
en même temps soutiennent que cette matière n'a point de force d'inertie ; établissent réellement l'existence du vide dont ils ne suppriment que le nom ; car puisqu'il n'y a aucune manière et aucune raison de distinguer une telle matière du vide, il est évident que ce n'est plus qu'une dispute de mots. Si malgré tout cela, il y a encore des personnes si fort attachées à la matière qu'elles veuillent croire qu'il n'est pas possible d'admettre un espace absolument vide de corps, voyons enfin où cette assertion les conduira.

Diront-ils que ce plein dans lequel ils imaginent que l'univers est construit, est un effet de la volonté de Dieu qui a tout disposé de cette manière afin de trouver pour les opérations de la nature une ressource toujours présente dans cette matière subtile qui pénètre et remplit tout ; quoique nous ayons déjà prouvé que l'on ne peut avancer cette proposition, puisqu'il est démontré par les phénomènes des Comètes qu'une telle matière ne peut avoir aucune efficacité ? Avanceront-ils que Dieu a voulu établir ce plein, pour une fin que nous ne connaissons pas, ce qui serait une autre absurdité, puisque l'on pourrait prouver par le même raisonnement toute autre disposition et tout autre mécanisme qu'il plairait d'imaginer pour expliquer le système de l'univers ? Oseraient-ils enfin nous assurer que ce plein universel n’est pas dépendant de la volonté de Dieu, mais qu'il doit son existence à une certaine nécessité de la nature ? Il faut donc qu'ils retombent dans toutes les impiétés de la plus méprisable de toutes les sectes, de ceux qui sont assez stupides pour croire que tout se fait au hasard, et non par une Providence souverainement intelligente ; de ces hommes qui s'imaginent que la matière a toujours existé nécessairement et en tout lieu, qu'elle est infinie et éternelle. Si on leur accordait ce principe, il s'ensuit aussi de là qu'elle doit être absolument uniforme et homogène dans toute son étendue ; car la variété des formes est directement opposée à la nécessité de l'existence : elle sera aussi par la même raison immobile ; car si elle se meut nécessairement vers un certain point de l'étendue, avec une certaine vitesse déterminée ; par une égale nécessité elle sera aussi en mouvement vers un autre point de l'étendue avec une vitesse différente ; mais il est évident qu'elle ne peut se mouvoir en même temps vers différents lieux et avec des vitesses différentes ; elle est donc nécessairement immobile. Donc il n'a pas pu résulter de cette matière un monde aussi beau et aussi admirable que le nôtre, par la variété des formes et des mouvements ; cet ou- 
vrage ne peut donc être qu'un effet de la volonté souverainement libre d'un Dieu qui prévoit tout et qui gouverne tout.

C'est là qu'il faut chercher la source et l'origine de toutes ces lois que nous appelons lois de la nature, dans lesquelles on retrouve à chaque instant les marques sensibles d'une intelligence infinie, sans jamais y découvrir le moindre trait qui puisse nous les faire regarder comme nécessaires. Se flatter de pouvoir découvrir les principes d'une vraie physique et les lois de la nature par la seule force de son génie, en fermant les yeux sur tout ce qui nous environne, pour ne consulter que la lumière d'une raison intérieure; c'est établir que le monde existe nécessairement, et que les lois dont il s'agit sont des suites immédiates de cette nécessité : ou si l'on est persuadé que cet Univers est l'ouvrage d'un Dieu ; c'est avoir assez d'orgueil pour imaginer qu'un être aussi petit, aussi faible que l'homme, connaît néanmoins avec évidence ce que Dieu pouvait faire de mieux. Toute Philosophie saine et véritable est uniquement appuyée sur les phénomènes. Si les mêmes phénomènes nous conduisent de gré ou de force à des principes dans lesquels on voit briller évidemment l'intelligence et le pouvoir absolu d'un Être souverainement sage et puissant; ce n'est pas une raison de les rejeter, parce qu'ils déplairont à quelques particuliers ; que ce soit pour ces gens-là des miracles ou des qualités occultes, on ne doit point leur imputer les noms que la malice peut leur donner ; à moins qu'on ne veuille nous avouer tout simplement que la philosophie doit être fondée sur l'Athéisme ; mais il ne faut pas altérer et corrompre la Philosophie pour des hommes de cette espèce ; l'ordre de la nature doit être aussi sacré qu'il est immuable.

Les gens de bien et les juges équitables dans cette matière regarderont certainement comme la plus excellente manière de traiter la Philosophie, celle qui est fondée sur les expériences et les observations. Nous ne pouvons exposer ici la gloire et l'éclat que cette nouvelle Philosophie reçoit de l'excellent Ouvrage, de notre illustre Auteur. Rien de plus juste que le respectueux étonnement avec lequel ceux qui ont approfondi ces matières ne cessent d'admirer la force et la grandeur de cet heureux génie occupé à résoudre les problèmes les plus difficiles, et si supérieur à tout ce que l'on pouvait attendre de l'esprit humain : il a, pour ainsi dire, déchiré le voile de la nature pour nous en découvrir les plus admirables mystères : il a mis sous nos yeux une exposition si élégante du système de l'univers, un ensemble si beau et si par- 
fait, qu'Alphonse ${ }^{3}$ lui-même n'aurait plus rien à désirer ni pour l'harmonie, ni pour la simplicité, si ce prince vivait encore. Nous pouvons maintenant contempler de plus près la majesté de la nature, jouir plus que jamais d'un spectacle si doux; adorer et servir avec plus d'ardeur le Maître et le Créateur de toutes choses, et c'est là le plus grand avantage que l'on puisse retirer de la Philosophie. Il faut être aveugle pour ne pas voir dans le meilleur et le plus sage de tous les ouvrages, la sagesse et la bonté infinie de celui qui en est l'auteur ; mais c'est le comble de la folie que de ne vouloir pas le reconnaître.

Ce grand Ouvrage de M. Newton sera donc un solide rempart que les impies et les athées ne pourront jamais renverser ; c'est là qu'il faut chercher des armes si l'on veut les combattre avec succès. Il y a déjà longtemps que cette importante vérité a été reconnue, par un illustre Professeur du Collège de la Trinité, M. Richard Bentley, qui fait à la fois la gloire de son siècle et l'ornement de notre Académie. Ce grand homme aussi recommandable par une vaste érudition que par la protection qu'il accorde à tous les Savants, est aussi le premier qui l'ait démontré avec autant de force que d'élégance dans ses discours académiques, si universellement estimés, et qui ont été publiés en latin et en anglais. Je me fais un plaisir de reconnaître ici combien je lui suis redevable à toutes sortes d'égards, et je ne doute point que le Lecteur ne soit pareillement disposé à lui payer le tribut de l'estime due à son savoir et à son mérite. Lié depuis longtemps d'une manière intime avec notre illustre Auteur; et d'ailleurs aussi sensible à cette gloire qu'à celle qu'il reçoit de ses ouvrages, qui font les délices de toutes les personnes lettrées, il a su rendre un service également important au nom de son ami et au progrès des sciences. Les exemplaires de la dernière édition des Principes étaient devenus très rares et se vendaient à un prix exorbitant. Il ne cessa de faire les plus vives instances à

Alphonse roi de Castille vivait vers le milieu du XIII ${ }^{\mathrm{e}}$ siècle : il donna des sommes prodigieuses pour faire construire de nouvelles tables astronomiques. On rapporte de lui un trait singulier qui revient à cet article. Lorsque les Astronomes qu'il avait choisis pour faire cet Ouvrage lui présentèrent leur système, qui se trouvait embarrassé d'une infinité de cercles qu'ils avoient cru nécessaires pour expliquer les différents mouvements des astres : Si Dieu dit ce Prince, m'eut consulté lorsqu'il créa l'Univers, tout aurait été dans un ordre meilleur et plus simple: Ironie adroite qui part moins d'un principe d'impiété, que d'un génie naturellement connaisseur, qui se doutait bien que le mécanisme l’Univers devait être beaucoup plus simple que celui qu'on lui proposait. 
M. Newton, et détermina enfin cet homme, aussi supérieur aux autres par sa modestie que par son savoir, à laisser paraître sous ses auspices et à ses dépens cette nouvelle édition que l'on a revue d'un bout à l'autre, et qui se trouve enrichie de diverses additions importantes que l'on y a faites ; enfin c'est par son crédit que je reçus dans le même temps une somme considérable qui me fut donnée, pour veiller à ce que cet Ouvrage fût exécuté avec tout le soin et toute la correction possible.

À Cambridge, le 12 Mai 1713.

nité, et

Roger Cotes, Associé du Collège de la Tripérimentale.

Professeur d'Astronomie et de Physique ex- 


\section{AVERTISSEMENT SUR LES PLANCHES DE CET OUVRAGE}

Comme on n'a pas voulu multiplier inutilement les Planches et les Figures, lorsqu'on trouvera une même Figure sous deux numéros, on regardera cette Figure comme si c’était deux figures séparées.

Les Planches qui étaient absolument nécessaires dans cet Ouvrage, et d'autres obstacles qu'on ne pouvait prévoir, ont empêché jusqu'ici la publication des Principes de Newton, qu'on se proposait de mettre en vente dès l'année 1756 .

\section{ERRATA ET SYMBOLES MATHÉMATIQUES}

Nous n'avons pas repris ici la liste des errata de l'édition de 1759, mais nous avons corrigé dans le texte de cette édition électronique toutes les erreurs qui y étaient signalées.

Nous avons cru bon d'homogénéiser l'orthographe des nom propres, et d'adopter l'orthographe actuelle. Par exemple, nous avons écrit Huygens (au lieu de Hughens), Thycho (à la place de Ticho) ou Viète (au lieu de Viet), etc.

Nous avons suivi, généralement, la nomenclature utilisée par $\mathrm{M}^{\text {me }}$ du Châtelet. Cependant, nous avons actualisé certains signes couramment utilisés en mathématiques au $18^{\mathrm{e}}$ siècle et qui n’ont plus cours aujourd'hui. Par exemple nous avons transcrit l'expression très fréquemment rencontrée $A: B:: C: D$ (A est à $B$ comme $C$ est à $D)$ par $A: B=C: D$, suivant en cela la publication The history of mathematical symbols, (http://www.unisanet.unisa.edu.au/07305/symbols.htm), du mathématicien D. Weaver.

Je tiens à remercier pour leur aide V. Simonet et E. Ressouche. J.-M. S. 


\title{
SUR LA PHYSIQUE DE NEWTON ${ }^{4}$
}

\author{
À MADAME \\ La marquise du Chastelet
}

Tu m’appelles à toi, vaste et puissant génie, Minerve de la France, immortelle Émilie.

Je m'éveille à ta voix, je marche à ta clarté, Sur les pas des vertus et de la vérité.

Je quitte Melpomène et les jeux du Théâtre, Ces combats, ces lauriers, dont je fus idolâtre.

De ces triomphes vains mon cœur n'est plus touché.

Que le jaloux Rufus, à la Terre attaché, Traîne au bord du tombeau la fureur insensée

D’enfermer dans un vers une fausse pensée ; Qu'il arme contre moi ses languissantes mains, Des traits qu'il destinait au reste des humains ; Que quatre fois par mois un ignorant Zoïle Élève en frémissant une voix imbécile ; Je n'entends point leurs cris que la haine a formés. Je ne vois point leurs pas dans la fange imprimés. Le charme tout-puissant de la Philosophie, Élève un esprit sage au-dessus de l'envie. Tranquille au haut des cieux, que Newton s'est soumis, Il ignore en effet s'il a des ennemis.

Je ne les connais plus. Déjà de la carrière L’auguste vérité vient m’ouvrir la barrière ; Déjà ces tourbillons, l’un par l'autre pressés, Se mouvant sans espace, et sans règles entassés, 
Ces fantômes savants à mes yeux disparaissent.

Un jour plus pur me luit ; les mouvements renaissent ;

L'espace, qui de Dieu contient l'immensité, Voit rouler dans son sein l’Univers limité,

Cet Univers si vaste à notre faible vue, Et qui n'est qu'un atome, un point dans l'étendue.

Dieu parle, et le chaos se dissipe à sa voix.

Vers un centre commun tout gravite à la fois.

Ce ressort si puissant, l'âme de la nature,

Était enseveli dans une nuit obscure.

Le compas de Newton, mesurant l'Univers, Lève enfin ce grand voile, et les Cieux sont ouverts.

Il découvre à mes yeux, par une main savante,

De l'astre des saisons la robe étincelante ;

L'émeraude, l'azur, le pourpre, le rubis, Sont l'immortel tissu dont brillent ses habits. Chacun de ses rayons dans sa substance pure, Porte en soi les couleurs dont se peint la nature, Et confondus ensemble ils éclairent nos yeux, Ils animent le monde, ils emplissent les Cieux.

Confidents du Très-haut, substances éternelles, Qui brûlez de ses feux, qui couvrez de vos ailes Le Trône où votre Maître est assis parmi vous, Parlez ; du grand Newton n'étiez-vous point jaloux ?

La mer entend sa voix. Je vois l'humide empire S'élever, s'avancer vers le Ciel qui l'attire :

Mais un pouvoir central arrête ses efforts ;

La mer tombe, s'affaisse, et roule vers ses bords.

Comètes, que l'on craint à l'égal du tonnerre,

Cessez d'épouvanter les peuples de la Terre ;

Dans une ellipse immense achevez votre cours ;

Remontez, descendez près de l'astre des jours ;

Lancez vos feux, volez ; et revenant sans cesse, Des mondes épuisés ranimez la vieillesse.

Et toi, sœur du Soleil, astre qui dans les Cieux

Des sages éblouis trompaient les faibles yeux, Newton de ta carrière a marqué les limites :

Marche, éclaire les nuits, tes bornes sont prescrites.

Terre, change de forme, et que la pesanteur, 
En abaissant le Pôle, élève l’Équateur.

Pôle, immobile aux yeux, si lent dans votre course, Fuyez le char glacé des sept Astres de l'Ourse : ${ }^{5}$

Embrassez dans le cours de vos longs mouvements

Deux cents siècles entiers par delà six mille ans.

Que ces objets sont beaux! Que notre âme épurée,

Vole à ces vérités dont elle est éclairée !

Oui, dans le sein de Dieu, loin de ce corps mortel,

L'esprit semble écouter la voix de l'Éternel.

Vous, à qui cette voix se fait si bien entendre,

Comment avez-vous pu, dans un âge encore tendre,

Malgré les vains plaisirs, ces écueils des beaux jours,

Prendre un vol si hardi, suivre un si vaste cours,

Marcher après Newton dans cette route obscure

Du labyrinthe immense où se perd la nature ?

Puissé-je auprès de vous, dans ce Temple écarté, Aux regards des Français montrer la Vérité, Tandis ${ }^{6}$ qu'Algaroti, sûr d'instruire et de plaire, Vers le Tibre étonné conduit cette Étrangère.

Que de nouvelles fleurs il orne ses attraits,

Le compas à la main j'en tracerai les traits ;

De mes crayons grossiers je peindrai l'immortelle ;

Cherchant à l'embellir, je la rendrais moins belle.

Elle est, ainsi que vous, noble, simple et sans fard, Au-dessus de l'éloge, au-dessus de mon art.

Table des matières

5 C’est la Période de la pression des Équinoxes, laquelle s'accomplit en vingtsix mille neuf cents ans, ou environ.

6 M. Algaroti, jeune Vénitien, faisait imprimer alors à Venise un Traité sur la Lumière, dans lequel il expliquait l'Attraction. Il y a eu sept éditions de son livre, lequel a été fort mal traduit en français. 


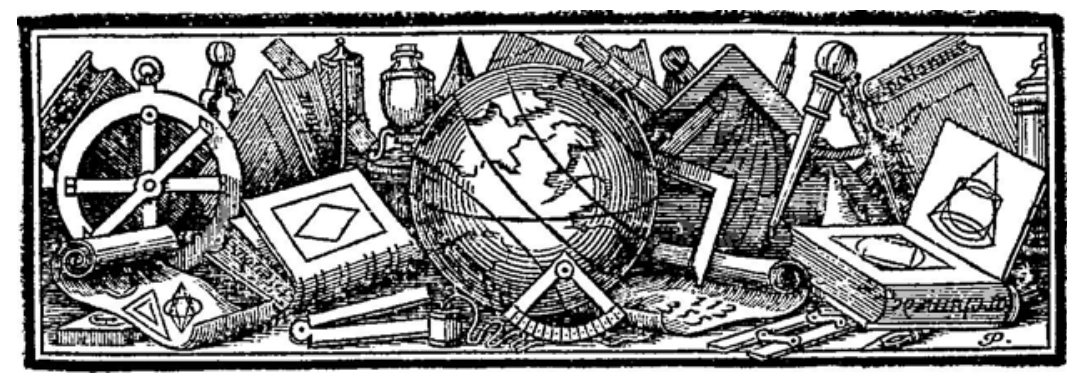

\section{PRINCIPES MATHÉMATIQUES}

\section{DE LA PHILOSOPHIE NATURELLE}

Table des matières

\section{DÉFINITIONS.}

\section{DÉFINITION PREMIÈRE.}

La quantité de matière se mesure par la densité et le volume pris ensemble.

L'air devenant d'une densité double est quadruple en quantité lorsque l'espace est double, et sextuple si l'espace est triple. On en peut dire autant de la neige et de la poudre condensées par la liquéfaction ou la compression, aussi bien que dans tous les corps condensés par quelque cause que ce puisse être.

Je ne fais point attention ici au milieu qui passe librement entre les parties des corps, supposé qu'un tel milieu existe. Je désigne la quantité de matière par les mots de corps ou de masse. Cette quantité se connaît par le poids des corps : car j'ai trouvé par des expériences très exactes sur les pendules, que les poids des corps sont proportionnels à leur masse ; je rapporterai ces expériences dans la suite. 


\section{DÉFINITION II.}

La quantité de mouvement est le produit de la masse par la vitesse.

Le mouvement total est la somme du mouvement de chacune des parties ; ainsi la quantité du mouvement est double dans un corps dont la masse est double, si la vitesse reste la même ; mais si on double la vitesse, la quantité du mouvement sera quadruple.

\section{DÉFINITION III.}

La force qui réside dans la matière (vis insita) est le pouvoir quelle a de résister. C'est par cette force que tout corps persévère de lui-même dans son état actuel de repos ou de mouvement uniforme en ligne droite.

Cette force est toujours proportionnelle à la quantité de matière des corps, et elle ne diffère de ce qu'on appelle l'inertie de la matière, que par la manière de la concevoir : car l'inertie est ce qui fait qu'on ne peut changer sans effort l'état actuel d'un corps, soit qu'il se meuve, soit qu'il soit en repos, ainsi on peut donner à la force qui réside dans les corps le nom très expressif de force d'inertie.

Le corps exerce cette force toutes les fois qu'il s'agit de changer son état actuel, et on peut la considérer alors sous deux différents aspects, ou comme résistante, ou comme impulsive : comme résistante, en tant que le corps s'oppose à la force qui tend à lui faire changer d'état ; comme impulsive, en tant que le même corps fait effort pour changer l'état de l’obstacle qui lui résiste.

On attribue communément la résistance aux corps en repos ; et la force impulsive à ceux qui se meuvent ; mais le mouvement et le repos, tels qu'on les conçoit communément, ne sont que respectifs : car les corps qu'on croit en repos ne sont pas toujours dans un repos absolu. 


\section{DÉFINITION IV.}

La force imprimée (vis impressa) est l'action par laquelle l'état du corps est changé, soit que cet état soit le repos, ou le mouvement uniforme en ligne droite.

Cette force consiste uniquement dans l'action, et elle ne subsiste plus dans le corps, dès que l'action vient à cesser. Mais le corps persévère par sa seule force d'inertie dans le nouvel état dans lequel il se trouve. La force imprimée peut avoir diverses origines, elle peut être produite par le choc, par la pression, et par la force centripète.

\section{DÉFINITION V.}

La force centripète est celle qui fait tendre les corps vers quelque point, comme vers un centre, soit qu'ils soient tirés ou poussés vers ce point, ou qu'ils y tendent d'une façon quelconque.

La gravité qui fait tendre tous les corps vers le centre de la Terre ; la force magnétique qui fait tendre le fer vers l'aimant, et la force, quelle qu'elle soit, qui retire à tout moment les planètes du mouvement rectiligne, et qui les fait circuler dans des courbes, sont des forces de ce genre.

La pierre qu'on fait tourner par le moyen d'une fronde, agit sur la main, en tendant la fronde, par un effort qui est d'autant plus grand qu'on la fait tourner plus vite, et elle s'échappe aussitôt qu'on ne la retient plus. La force exercée par la main pour retenir la pierre, laquelle est égale et contraire à la force par laquelle la pierre tend la fronde, étant donc toujours dirigée vers la main, centre du cercle décrit, est celle que j'appelle force centripète. Il en est de même de tous les corps qui se meuvent en rond, ils font tous effort pour s'éloigner du centre de leur révolution; et sans le secours de quelque force qui s'oppose à cet effort et qui les retient dans leurs orbes, c'est-à-dire, de quelque force centripète, ils s'en iraient en ligne droite d'un mouvement uniforme.

Un projectile ne retomberait point vers la Terre, s'il n'était point animé par la force de la gravité, mais il s’en irait en ligne droite dans les cieux avec un mouvement uniforme, si la résistance de l'air était 
nulle. C’est donc par sa gravité qu'il est retiré de la ligne droite, et qu'il s’infléchit sans cesse vers la Terre; et il s'infléchit plus ou moins, selon sa gravité et la vitesse de son mouvement. Moins la gravité du projectile sera grande par rapport à sa quantité de matière, plus il aura de vitesse, moins il s'éloignera de la ligne droite, et plus il ira loin avant de retomber sur la Terre.

Ainsi, si un boulet de canon était tiré horizontalement du haut d'une montagne, avec une vitesse capable de lui faire parcourir un espace de deux lieues avant de retomber sur la Terre : avec une vitesse double, il n'y retomberait qu'après avoir parcouru à peu près quatre lieues, et avec une vitesse décuple, il irait dix fois plus loin ; (pourvu qu'on n'ait point d'égard à la résistance de l'air,) et en augmentant la vitesse de ce corps, on augmenterait à volonté le chemin qu'il parcourrait avant de retomber sur la Terre, et on diminuerait la courbure de la ligne qu'il décrirait ; en sorte qu'il pourrait ne retomber sur la Terre qu'à la distance de 10, de 30, ou de 90 degrés; ou qu'enfin il pourrait circuler autour, sans y retomber jamais, et même s'en aller en ligne droite à l'infini dans le ciel.

Or, par la même raison qu'un projectile pourrait tourner autour de la Terre par la force de la gravité, il se peut faire que la lune par la force de sa gravité, (supposé qu'elle gravite) ou par quelqu'autre force qui la porte vers la Terre, soit détournée à tout moment de la ligne droite pour s'approcher de la Terre, et qu'elle soit contrainte à circuler dans une courbe, et sans une telle force, la lune ne pourrait être retenue dans son orbite.

Si cette force était moindre qu'il ne convient, elle ne retirerait pas assez la Lune de la ligne droite, et si elle était plus grande, elle l'en retirerait trop, et elle la tirerait de son orbe vers la Terre. La quantité de cette force doit donc être donnée ; et c'est aux Mathématiciens à trouver la force centripète nécessaire pour faire circuler un corps dans une orbite donnée, et à déterminer réciproquement la courbe dans laquelle un corps doit circuler par une force centripète donnée, en partant d'un lieu quelconque donné, avec une vitesse donnée.

La quantité de la force centripète peut être considérée comme $a b$ solue, accélératrice et motrice. 


\section{DÉFINITION VI.}

La quantité, absolue de la force centripète est plus grande ou moindre, selon l'efficacité de la cause qui la propage du centre.

C'est ainsi que la force magnétique est plus grande dans un aimant que dans un autre, suivant la grandeur de la pierre, et l'intensité de sa vertu.

\section{DÉFINITION VII.}

La quantité accélératrice de la force centripète est proportionnelle à la vitesse qu'elle produit dans un temps donné.

La force magnétique du même aimant est plus grande à une moindre distance, qu'à une plus grande. La force de la gravité est plus grande dans les plaines, et moindre sur le sommet des hautes montagnes, et doit être encore moindre (comme on le prouvera dans la suite) à de plus grandes distances de la Terre, et à des distances égales, elle est la même de tous côtés ; c'est pourquoi elle accélère également tous les corps qui tombent, soit qu'ils soient légers ou pesants, grands ou petits, abstraction faite de la résistance de l'air.

\section{DÉFINITION VIII.}

La quantité motrice de la force centripète est proportionnelle au mouvement qu'elle produit dans un temps donné.

Le poids des corps est d'autant plus grand, qu'ils ont plus de masse ; et le même corps pèse plus près de la surface de la Terre, que s’il était transporté dans le ciel. La quantité motrice de la force centripète est la force totale avec laquelle le corps tend vers le centre, et proprement son poids ; et on peut toujours la connaître en connaissant la force contraire et égale qui peut empêcher le corps de descendre.

J'ai appelé ces différentes quantités de la force centripète, motrices, accélératrices, et absolues, afin d'être plus court.

On peut, pour les distinguer, les rapporter aux corps qui sont attirés vers un centre, aux lieux de ces corps, et au centre des forces. 
On peut rapporter la force centripète motrice au corps, en la considérant comme l'effort que fait le corps entier pour s'approcher du centre, lequel effort est composé de celui de toutes ses parties.

La force centripète accélératrice peut se rapporter au lieu du corps, en considérant cette force en tant qu'elle se répand du centre dans tous les lieux qui l'environnent, pour mouvoir les corps qui s'y rencontrent.

Enfin on rapporte la force centripète absolue au centre, comme à une certaine cause sans laquelle les forces motrices ne se propageraient point dans tous les lieux qui entourent le centre ; soit que cette cause soit un corps central quelconque, (comme l'aimant dans le centre de la force magnétique, et la Terre dans le centre de la force gravitante), soit que ce soit quelque autre cause qu'on n'aperçoit pas. Cette façon de considérer la force centripète est purement mathématique : et je ne prétends point en donner la cause physique.

La force centripète accélératrice est donc à la force centripète motrice, ce que la vitesse est au mouvement ; car de même que la quantité de mouvement est le produit de la masse par la vitesse, la quantité de la force centripète motrice est le produit de la force centripète accélératrice par la masse ; car la somme de toutes les actions de la force centripète accélératrice sur chaque particule du corps est la force centripète motrice du corps entier. Donc à la surface de la Terre où la force accélératrice de la gravité est la même sur tous les corps, la gravité motrice ou le poids des corps est proportionnel à leur masse ; et si on était placé dans des régions où la force accélératrice diminuait, le poids des corps y diminuerait pareillement ; ainsi il est toujours comme le produit de la masse par la force centripète accélératrice. Dans les régions où la force centripète accélératrice serait deux fois moindre, le poids d'un corps sous double ou sous triple serait quatre fois ou six fois moindre.

Au reste, je prends ici dans le même sens les attractions et les impulsions accélératrices et motrices, et je me sers indifféremment des mots d'impulsion, d'attraction, ou de propension quelconque vers un centre : car je considère ces forces mathématiquement et non physiquement ; ainsi le Lecteur doit bien se garder de croire que j'aie voulu désigner par ces mots une espèce d'action, de cause ou de raison phy- 
sique, et lorsque je dis que les centres attirent, lorsque je parle de leurs forces, il ne doit pas penser que j'aie voulu attribuer aucune force réelle à ces centres que je considère comme des points mathématiques.

\section{SCHOLIE.}

Je viens de faire voir le sens que je donne dans cet Ouvrage à des termes qui ne sont pas communément usités. Quant à ceux de temps, d'espace, de lieu et de mouvement, ils sont connus de tout le monde ; mais il faut remarquer que pour n'avoir considéré ces quantités que par leurs relations à des choses sensibles, on est tombé dans plusieurs erreurs.

Pour les éviter, il faut distinguer le temps, l'espace, le lieu, et le mouvement, en absolus et relatifs, vrais et apparents, mathématiques et vulgaires.

I. Le temps absolu, vrai et mathématique, sans relation à rien d'extérieur, coule uniformément, et s'appelle durée. Le temps relatif, apparent et vulgaire, est cette mesure sensible et externe d'une partie de durée quelconque (égale ou inégale) prise du mouvement : telles sont les mesures d'heures, de jours, de mois, et c'est ce dont on se sert ordinairement à la place du temps vrai.

II. L'espace absolu, sans relation aux choses externes, demeure toujours similaire et immobile.

L'espace relatif est cette mesure ou dimension mobile de l'espace absolu, laquelle tombe sous nos sens par sa relation aux corps, et que le vulgaire confond avec l'espace immobile. C'est ainsi, par exemple, qu'un espace, pris au-dedans de la Terre ou dans le ciel, est déterminé par la situation qu'il a à l'égard de la Terre.

L'espace absolu et l'espace relatif sont les mêmes d'espèce et de grandeur ; mais ils ne le sont pas toujours de nombre ; car, par exemple, lorsque la Terre change de place dans l'espace, l'espace qui contient notre air demeure le même par rapport à la Terre, quoique l'air occupe nécessairement les différentes parties de l'espace dans lesquelles il passe, et qu'il en change réellement sans cesse. 
III. Le lieu est la partie de l'espace occupée par un corps, et par rapport à l'espace, il est ou relatif ou absolu.

Je dis que le lieu est une partie de l'espace, et non pas simplement la situation du corps, ou la superficie qui l'entoure : car les solides égaux ont toujours des lieux égaux, quoique leurs superficies soient souvent inégales, à cause de la dissemblance de leurs formes, les situations, à parler exactement, n’ont point de quantité, ce sont plutôt des affections des lieux, que des lieux proprement dits.

De même que le mouvement ou la translation du tout hors de son lieu est la somme des mouvements ou des translations des parties hors du leur ; ainsi le lieu du tout est la somme des lieux de toutes les parties, et ce lieu doit être interne, et être dans tout le corps entier (et propterea internus et in corpore toto).

IV. Le mouvement absolu est la translation des corps d'un lieu absolu dans un autre lieu absolu, et le mouvement relatif est la translation d'un lieu relatif dans un autre lieu relatif ; ainsi dans un vaisseau poussé par le vent, le lieu relatif d'un corps est la partie du vaisseau dans laquelle ce corps se trouve, ou l'espace qu'il occupe dans la cavité du vaisseau ; et cet espace se meut avec le vaisseau ; et le repos relatif de ce corps est sa permanence dans la même partie de la cavité du vaisseau. Mais le repos vrai du corps est sa permanence dans la partie de l'espace immobile, où l'on suppose que se meut le vaisseau et tout ce qu'il contient. Ainsi, si la Terre était en repos, le corps qui est dans un repos relatif dans le vaisseau aurait un mouvement vrai et absolu, dont la vitesse serait égale à celle qui emporte le vaisseau sur la surface de la Terre ; mais la Terre se mouvant dans l'espace, le mouvement vrai et absolu de ce corps est composé du mouvement vrai de la Terre dans l'espace immobile, et du mouvement relatif du vaisseau sur la surface de la Terre ; et si le corps avait un mouvement relatif dans le vaisseau, son mouvement vrai et absolu serait composé de son mouvement relatif dans le vaisseau, du mouvement relatif du vaisseau sur la Terre, et du mouvement vrai de la Terre dans l'espace absolu. Quant au mouvement relatif de ce corps sur la Terre, il serait formé dans ce cas de son mouvement relatif dans le vaisseau, et du mouvement relatif du vaisseau sur la Terre. En sorte que si la partie de la Terre où se trouve ce vaisseau avait un mouvement vrai vers l'orient, avec une vitesse divisée en 10010 parties : que le vaisseau fût empor- 
té vers l'occident avec 10 parties de cette vitesse ; et que le Pilote se promenât dans le vaisseau vers l'orient, avec une partie, de cette même vitesse: ce pilote aurait un mouvement réel et absolu dans l'espace immobile, avec 10001 parties de vitesse vers l'orient, et un mouvement relatif sur la Terre vers l'occident avec 9 parties de vitesse.

On distingue en astronomie le temps absolu du temps relatif par l'équation du temps. Car les jours naturels sont inégaux, quoiqu'on les prenne communément pour une mesure égale du temps ; et les Astronomes corrigent cette inégalité, afin de mesurer les mouvements célestes par un temps plus exact.

Il est très possible qu'il n'y ait point de mouvement parfaitement égal, qui puisse servir de mesure exacte du temps ; car tous les mouvements peuvent être accélérés et retardés, mais le temps absolu doit toujours couler de la même manière.

La durée ou la persévérance des choses est donc la même, soit que les mouvements soient prompts, soit qu'ils soient lents, et elle serait encore la même, quand il n'y aurait aucun mouvement, ainsi il faut bien distinguer le temps de ses mesures sensibles, et c'est ce qu'on fait par l'équation astronomique. La nécessité de cette équation dans la détermination des phénomènes se prouve assez par l'expérience des horloges à pendule, et par les observations des Éclipses des satellites de Jupiter.

L'ordre des parties de l'espace est aussi immuable que celui des parties du temps ; car si les parties de l'espace sortaient de leur lieu, ce serait, si l'on peut s'exprimer ainsi, sortir d'elles-mêmes. Les temps et les espaces n'ont pas d'autres lieux qu'eux-mêmes, et ils sont les lieux de toutes les choses. Tout est dans le temps, quant à l'ordre de la succession : tout est dans l'espace, quant à l'ordre de la situation. C'est là ce qui détermine leur essence, et il serait absurde que les lieux primordiaux se mussent. Ces lieux sont donc les lieux absolus, et la seule translation de ces lieux fait les mouvements absolus.

Comme les parties de l'espace ne peuvent être vues ni distinguées les unes des autres par nos sens, nous y suppléons par des mesures sensibles. Ainsi nous déterminons les lieux par les positions et les distances à quelque corps que nous regardons comme immobile, et nous 
mesurons ensuite les mouvements des corps par rapport à ces lieux ainsi déterminés : nous nous servons donc des lieux et des mouvements relatifs à la place des lieux et des mouvements absolus; et il est à propos d'en user ainsi dans la vie civile : mais dans les matières philosophiques, il faut faire abstraction des sens, car il se peut faire qu'il n'y ait aucun corps véritablement en repos, auquel on puisse rapporter les lieux et les mouvements.

Le repos et le mouvement relatifs et absolus sont distingués par leurs propriétés, leurs causes et leurs effets. La propriété du repos est que les corps véritablement en repos y sont les uns à l'égard des autres. Ainsi, quoiqu'il soit possible qu'il y ait quelque corps dans la région des fixes, ou beaucoup au-delà, qui soit dans un repos absolu, comme on ne peut pas connaître par la situation qu'ont entre eux les corps d'ici-bas, si quelques uns de ces corps conservent ou non sa situation par rapport à ce corps éloigné, on ne saurait déterminer, par le moyen de leur situation que ces corps ont entre eux, s’ils sont véritablement en repos.

La propriété du mouvement est que les parties qui conservent des positions données par rapport aux touts participent aux mouvements de ces touts ; car si un corps se meut autour d'un axe, toutes ses parties font effort pour s'éloigner de cet axe, et s'il a un mouvement progressif, son mouvement total est la somme des mouvements de toutes ses parties. De cette propriété il suit que si un corps se meut, les corps qu'il contient, et qui sont par rapport à lui dans un repos relatif, se meuvent aussi ; et par conséquent le mouvement vrai et absolu ne saurait être défini par la translation du voisinage des corps extérieurs, que l'on considère comme en repos. Il faut que les corps extérieurs soient non seulement regardés comme en repos, mais qu'ils y soient véritablement : autrement les corps qu'ils renferment, outre leur translation du voisinage des ambiants, participeront encore au mouvement vrai des ambiants, et s'ils ne changeaient point de position par rapport aux parties des ambiants, ils ne seraient pas pour cela véritablement en repos ; mais ils seraient seulement considérés comme en repos. Les corps ambiants sont à ceux qu'ils contiennent, comme toutes les parties extérieures d'un corps sont à toutes ses parties intérieures, ou comme l'écorce est au noyau. Or l'écorce étant mue, le noyau se meut aussi, quoiqu'il ne change point sa situation par rapport aux parties de l'écorce qui l'environnent. 
Il suit de cette propriété du mouvement qu'un lieu étant mû, tout ce qu'il contient se meut aussi, et par conséquent qu'un corps qui se meut dans un lieu mobile, participe au mouvement de ce lieu. Tous les mouvements qui s'exécutent dans des lieux mobiles ne sont donc que les parties des mouvements entiers et absolus. Le mouvement entier et absolu d'un corps est composé du mouvement de ce corps dans le lieu où l'on le suppose, du mouvement de ce lieu dans le lieu où il est placé lui-même, et ainsi de suite, jusqu'à ce qu'on arrive à un lieu immobile, comme dans l'exemple du Pilote dont on a parlé ci-dessus. Ainsi les mouvements entiers et absolus ne peuvent se déterminer qu'en les considérant dans un lieu immobile : et c'est pourquoi j'ai rapporté cidessus les mouvements absolus à un lieu immobile, et les mouvements relatifs à un lieu mobile. Il n'y a de lieux immobiles que ceux qui conservent à l'infini dans tous les sens leurs situations respectives ; et ce sont ces lieux qui constituent l'espace que j'appelle immobile.

Les causes par lesquelles on peut distinguer le mouvement vrai du mouvement relatif sont les forces imprimées dans les corps pour leur donner le mouvement : car le mouvement vrai d'un corps ne peut être produit ni changé que par des forces imprimées à ce corps même ; au lieu que son mouvement relatif peut être produit et changé, sans qu'il éprouve l'action d'aucune force : il suffit qu'il y ait des forces qui agissent sur les corps par rapport auxquels on le considère, puisque ces corps étant mus, la relation dans laquelle consiste le repos ou le mouvement relatif change, de même, le mouvement absolu d'un corps peut changer, sans que son mouvement relatif change ; car si les forces qui agissent sur ce corps agissaient en même temps sur ceux par rapport auxquels on le considère, et en telle sorte que les relations restassent toujours les mêmes, le mouvement relatif, qui n'est autre chose que ces relations, ne changerait point. Ainsi le mouvement relatif peut changer, tandis que le mouvement vrai et absolu reste le même, et il peut se conserver aussi, quoique le mouvement absolu change ; il est donc sûr que le mouvement absolu ne consiste point dans ces sortes de relations.

Les effets par lesquels on peut distinguer le mouvement absolu du mouvement relatif, sont les forces qu'ont les corps qui tournent pour s'éloigner de l'axe de leur mouvement ; car dans le mouvement circulaire purement relatif, ces forces sont nulles, et dans le mouvement 
circulaire vrai et absolu elles sont plus ou moins grandes, selon la quantité du mouvement.

Si on fait tourner en rond un vase attaché à une corde jusqu'à ce que la corde, à force d'être torse, devienne en quelque sorte inflexible ; si on met ensuite de l'eau dans ce vase, et qu'après avoir laissé prendre à l'eau et au vase l'état de repos, on donne à la corde la liberté de se détortiller, le vase acquerra par ce moyen un mouvement qui se conservera très longtemps : au commencement de ce mouvement la superficie de l'eau contenue dans le vase restera plane, ainsi qu'elle l'était avant que la corde se détortillât ; mais ensuite le mouvement du vase se communiquant peu à peu à l'eau qu'il contient, cette eau commencera à tourner, à s'élever vers les bords, et à devenir concave, comme je l'ai éprouvé, et son mouvement s'augmentant, les bords de cette eau s'élèveront de plus en plus, jusqu'à ce que les révolutions s'achevant dans des temps égaux à ceux dans lesquels le vase fait un tour entier, l'eau sera dans un repos relatif par rapport à ce vase. L'ascension de l'eau vers les bords du vase marque l'effort qu'elle fait pour s'éloigner du centre de son mouvement, et on peut connaître et mesurer par cet effort le mouvement circulaire vrai et absolu de cette eau, lequel est entièrement contraire à son mouvement relatif ; car dans le commencement où le mouvement relatif de l'eau dans le vase était le plus grand, ce mouvement n'excitait en elle aucun effort pour s'éloigner de l'axe de son mouvement: l'eau ne s'élevait point vers les bords du vase, mais elle demeurait plane, et par conséquent elle n'avait pas encore de mouvement circulaire vrai et absolu : lorsque ensuite le mouvement relatif de l'eau vint à diminuer, l'ascension de l'eau vers les bords du vase marquait l'effort qu'elle faisait pour s'éloigner de l'axe de son mouvement ; et cet effort, qui allait toujours en augmentant, indiquait l'augmentation de son mouvement circulaire vrai. Enfin ce mouvement vrai fut le plus grand, lorsque l'eau fut dans un repos relatif dans le vase. L'effort que faisait l'eau pour s'éloigner de l'axe de son mouvement, ne dépendait donc point de sa translation du voisinage des corps ambiants, et par conséquent le mouvement circulaire vrai ne peut se déterminer par de telles translations.

Le mouvement vrai circulaire de tout corps qui tourne est unique, et il répond à un seul effort qui est la mesure naturelle et exacte ; mais les mouvements relatifs sont variés à l'infini, selon toutes les relations aux corps extérieurs ; et tous ces mouvements, qui ne sont que des re- 
lations, n’ont aucun effet réel, qu'en tant qu'ils participent du mouvement vrai et unique. De-là, il suit que dans le système de ceux qui prétendent que nos cieux tournent au-dessous des cieux des Étoiles fixes, et qu'ils emportent les Planètes par leurs mouvements : toutes les parties des cieux, et les Planètes qui sont en repos par rapport aux cieux qui les environnent se meuvent réellement ; car elles changent leur position entre elles (au contraire de ce qui arrive aux corps qui sont dans un repos absolu) et étant transportées avec les cieux qui les entourent, elles font effort, ainsi que les parties des touts qui tournent, pour s’éloigner de l'axe du mouvement.

Les quantités relatives ne sont donc pas les véritables quantités dont elles portent le nom, mais ce sont les mesures sensibles, (exactes ou non exactes) que l'on emploie ordinairement pour les mesurer. Or comme la signification des mots doit répondre à l'usage qu'on en fait, on aurait tort si on entendait par les mots de temps, d'espace, de lieu et de mouvement, autre chose que les mesures sensibles de ces quantités, excepté dans le langage purement mathématique. Lorsqu'on trouve donc ces termes dans l'Écriture, ce serait faire violence au texte sacré, si au lieu de les prendre pour les quantités qui leur servent de mesures sensibles, on les prenait pour les véritables quantités absolues, ce serait de même aller contre le but de la Philosophie et des Mathématiques, de confondre ces mêmes mesures sensibles ou quantités relatives avec les quantités absolues qu'elles mesurent.

Il faut avouer qu'il est très difficile de connaître les mouvements vrais de chaque corps, et de les distinguer actuellement des mouvements apparents, parce que les parties de l'espace immobile dans lesquelles s'exécutent les mouvements vrais, ne tombent pas sous nos sens. Cependant il ne faut pas en désespérer entièrement, car on peut se servir, pour y parvenir, tant des mouvements apparents, qui sont les différences des mouvements vrais, que des forces qui sont les causes et les effets des mouvements vrais. Si, par exemple, deux globes attachés l'un à l'autre par le moyen d'un fil de longueur donnée viennent à tourner autour de leur centre commun de gravité, la tension du fil fera connaître l'effort qu'ils font pour s'écarter du centre du mouvement, et donnera par ce moyen la quantité du mouvement circulaire. Ensuite, si en frappant ces deux globes en même temps, dans des sens opposés, et avec des forces égales, on augmente ou on diminue le mouvement circulaire, on connaîtra par l'augmentation ou la diminu- 
tion de la tension du fil, l'augmentation ou la diminution du mouvement ; et enfin on trouvera par ce moyen les côtés de ces globes où les forces doivent être imprimées pour augmenter le plus qu'il est possible le mouvement, c'est-à-dire, les côtés qui se meuvent parallèlement au fil, et qui suivent son mouvement, connaissant donc ces côtés et leurs opposés qui précèdent le mouvement du fil, on aura la détermination du mouvement.

On parviendrait de même à connaître la quantité et la détermination de ce mouvement circulaire dans un vide quelconque immense, où il n'y aurait rien d'extérieur ni de sensible à quoi on pût rapporter le mouvement de ces globes.

Si dans cet espace il se trouvait quelques autres corps très éloignés qui conservassent toujours entre eux une position donnée, tels que sont les étoiles fixes, on ne pourrait savoir par la translation relative des globes, par rapport à ces corps, s’il faudrait attribuer le mouvement aux globes, ou s’il le faudrait supposer dans ces corps ; mais si en faisant attention au fil qui joint les globes, on trouvait sa tension telle que le mouvement des globes le requiert ; alors non seulement on verrait avec certitude que ce sont les globes qui se meuvent, et que les autres corps sont en repos ; mais on aurait la détermination du mouvement de ces globes par leurs translations relatives à l'égard des corps.

On fera voir plus amplement dans la suite comment les mouvements vrais peuvent se connaître par leurs causes, leurs effets, et leurs différences apparentes, et comment on peut connaître au contraire par les mouvements vrais ou apparents leurs causes et leurs effets, et c'est principalement dans cette vue qu'on a composé cet Ouvrage. 


\section{AXIOMES \\ OU LOIS DU MOUVEMENT.}

\section{PREMIÈRE LOI.}

Tout corps persévère dans l'état de repos ou de mouvement uniforme en ligne droite dans lequel il se trouve, à moins que quelque force n'agisse sur lui, et ne le contraigne à changer d'état.

Les projectiles par eux-mêmes persévèrent dans leurs mouvements, mais la résistance de l'air les retarde, et la force de la gravité les porte vers la Terre. Une toupie, dont les parties se détournent continuellement les unes les autres de la ligne droite par leur cohérence réciproque, ne cesse de tourner, que parce que la résistance de l'air la retarde peu à peu. Les planètes et les comètes qui sont de plus grandes masses, et qui se meuvent dans des espaces moins résistants, conservent plus longtemps leurs mouvements progressifs et circulaires.

\section{II $^{\mathrm{e}}$ LOI.}

Les changements qui arrivent dans le mouvement sont proportionnels à la force motrice, et se font dans la ligne droite dans laquelle cette force a été imprimée.

Si une force produit un mouvement quelconque, une force double de cette première produira un mouvement double, et une force triple un mouvement triple, soit qu'elle ait été imprimée en un seul coup, soit qu'elle l'ait été peu à peu et successivement, et ce mouvement, étant toujours déterminé du même côté que la force génératrice, sera ajouté au mouvement que le corps est supposé avoir déjà, s’il conspire avec lui ; ou en sera, retranché, s'il lui est contraire, ou bien sera retranché ou ajouté en partie, s'il lui est oblique ; et de ces deux mouvements il s'en formera un seul, dont la détermination sera composée des deux premières. 


\section{III $^{\mathrm{e}}$ LOI.}

L'action est toujours égale et opposée à la réaction ; c'està-dire, que les actions de deux corps l'un sur l'autre sont toujours égales, et dans des directions contraires.

Tout corps qui presse ou tire un autre corps est en même temps tiré ou pressé lui-même par cet autre corps. Si on presse une pierre avec le doigt, le doigt est pressé en même temps par la pierre. Si un cheval tire une pierre par le moyen d'une corde, il est également tiré par la pierre : car la corde qui les joint et qui est tendue des deux côtés, fait un effort égal pour tirer la pierre vers le cheval, et le cheval vers la pierre ; et cet effort s'oppose autant au mouvement de l'un, qu'il excite le mouvement de l'autre.

Si un corps en frappe un autre, et qu'il change son mouvement, de quelque façon que ce soit, le mouvement du corps choquant sera aussi changé de la même quantité et dans une direction contraire par la force du corps choqué, à cause de l'égalité de leur pression mutuelle.

Par ces actions mutuelles, il se fait des changements égaux, non pas de vitesse, mais de mouvement, pourvu qu'il ne s'y mêle aucune cause étrangère ; car les changements de vitesse qui se font de la même manière dans des directions contraires doivent être réciproquement proportionnels aux masses, à cause que les changements de mouvement sont égaux. Cette loi a lieu aussi dans les attractions, comme je le prouverai dans le scholie suivant.

\section{COROLLAIRE I.}

Un corps poussé par deux forces parcourt, par leurs actions réunies, la diagonale d'un parallélogramme dans le même temps, dans lequel il aurait parcouru ses côtés séparément.

\section{(Fig. 1)}

Si le corps, pendant un temps donné, eut été transporté de $A$ en $B$, d'un mouvement uniforme par la seule force $M$ imprimée en $A$; et que par la seule force $N$, imprimée dans le même lieu $A$, il eut été transporté de $A$ en $C$, le corps par ces deux forces réunies sera transporté dans le même temps dans la diagonale $A D$ du parallélogramme $A B C D$; car 
puisque la force $N$ agit selon la ligne $A C$ parallèle à $B D$, cette force, selon la seconde loi du mouvement, ne changera rien à la vitesse avec laquelle ce corps s'approche de cette ligne $B D$, par l'autre force $M$. Le corps s'approchera donc de la ligne $B D$ dans le même temps, soit que la force $N$ lui soit imprimée, soit qu'elle ne le soit pas ; ainsi à la fin de ce temps il sera dans quelque point de cette ligne $B D$. On prouvera, de la même manière qu'à la fin de ce même temps le corps sera dans un point quelconque de la ligne $C D$. Donc il sera nécessairement dans le point d'intersection $D$ de ces deux lignes, et par la première loi il ira d'un mouvement rectiligne de $A$ en $D$.

\section{COROLlaire II.}

D'où l'on voit qu'une force directe $A D$ est composée des forces obliques quelconques $A B$ et $B D$, et réciproquement qu'elle peut toujours se résoudre dans les forces obliques quelconques $A B$ et $B D$. Cette résolution et cette composition des forces se trouve confirmée à tout moment dans la mécanique.

(Fig. 2)

Supposons que du centre $O$ d'une roue partent des rayons inégaux $O M, O N$, qui soutiennent par des fils $M A, N P$ des poids $A$ et $P$, et qu'on cherche les forces de ces poids pour faire tourner cette roue.

On mènera d'abord par le centre $O$ la droite $K O L$ perpendiculaire en $K$ et en $L$ aux fils $M A, N P$, et du centre $O$ et de l'intervalle $O L$, le plus grand des intervalles $O K, O L$ on décrira un cercle. On tirera ensuite par le centre $O$, et par l'intersection $D$ de ce cercle avec le fil $M A$ la droite $O D$ à laquelle on mènera par $A$ la parallèle $A C$, terminée en $C$ par la droite $D C$, qui lui est perpendiculaire. Cela posé, comme il est indifférent que les points $K, L, D$, des fils soient attachés ou non au plan de la roue, les poids feront le même effet, soit qu'ils soient attachés aux points $K$ et $L$, soit qu'ils soient attachés aux points $D$ et $L$.

Soit donc exprimée la force totale du corps $A$ par la ligne $A D$, et soit cette force décomposée dans les deux forces $A C$, et $C D$, la première $A C$ tirant le rayon $O D$ dans sa direction, ne contribue point au mouvement de la roue, mais la seconde $D C$ tirant le rayon $O D$ perpendiculairement, fait le même effet que si elle tirait perpendiculaire- 
ment le rayon $O L$ égal à $O D$, c'est-à-dire qu'elle sera équivalente au poids $P$, pourvu que ce poids soit au poids $A$, comme la force $D C$ est à la force $D A$, ou, ce qui revient au même (à cause des triangles semblables $A D C, D O K$ ) comme $O K$ à $O D$ ou $O L$ : donc si les poids $A$ et $P$ sont pris dans la raison renversée des rayons $O K, O L$, auxquels ils sont appliqués, ils seront en équilibre, ce qui est la propriété si connue du levier, de la balance, et du treuil. Si l'un des poids est à l'autre dans une plus grande raison, sa force en sera d'autant plus grande pour mouvoir la roue.

Supposons présentement que le poids $p$ égal au poids $P$, soit en partie soutenu par le fil $N p$, et en partie par le plan $p G$, on mènera $p H$ et $N H$, la première perpendiculaire à l'horizon, et l'autre au plan $p G$, et prenant $p H$ pour exprimer la force avec laquelle le corps $p$ tend en en bas, on décomposera cette force dans les deux $\mathrm{pH}$ et $\mathrm{NH}$. Imaginant ensuite que le poids $p$, au lieu d'être attaché au fil $N p$, fut arrêté par un plan $p Q$ perpendiculaire à la direction $N p$, et coupant le plan $p G$, dans une ligne parallèle à l'horizon, il est clair que les forces avec lesquelles le corps presserait les plans $p Q, p G$, qui le retiendraient dans cette supposition, seraient exprimées, la première par $p N$, et la seconde par $H N$. Donc en supprimant le plan $p Q$, et laissant le fil $N p$ qui fait absolument le même effet, la tension de ce fil sera la même force $p N$ avec laquelle le plan $p Q$ était pressé.

Ainsi la tension du fil, lorsqu'il est dans la situation oblique $p N$, est à la tension du même fil, lorsqu'il a, comme dans le cas précédent, la situation perpendiculaire $P N$, comme $p N$ à $p H$. C'est pourquoi si le poids $p$ est au poids $A$ dans la raison composée de la raison réciproque des moindres distances du centre de la roue aux fils $p N$ et $A M$, et de la raison directe de $p H$ à $p N$; ces poids auront une égale force pour faire mouvoir la roue, et seront par conséquent en équilibre, ce dont tout le monde peut reconnaître la vérité.

Le poids $p$, en s'appuyant sur ces deux plans obliques, est dans le même cas qu'un coin entre les deux surfaces internes du corps qu'il fend : et on peut connaître par là, les forces du coin et du marteau : puisqu'en effet la force avec laquelle le corps $p$, presse le plan $p Q$, est à la force avec laquelle ce même corps est poussé vers ces plans, suivant la ligne perpendiculaire $p H$, par la force de sa gravité ou par les 
coups du marteau, comme $p N$ à $p H$; et à la force par laquelle il presse l'autre plan $p G$, comme $p N$ à $H N$.

On peut par une semblable décomposition des forces trouver la force de la vis ; car la vis n'est autre chose qu'un coin mû par un levier, ce qui fait voir la fécondité de ce Corollaire, et fournit de nouvelles preuves de la vérité ; il peut servir de base à toute la mécanique dans laquelle on a employé jusqu’à présent tant de différents principes.

On en tire aisément, par exemple, les forces de toutes les machines composées de roues, de tambours, de poulies, de leviers, de cordes tendues, de poids montants directement ou obliquement, et enfin de toutes les puissances dont les machines sont ordinairement composées ; on en tirerait aussi les forces nécessaires aux tendons pour mouvoir les membres des animaux.

\section{COROLLAIRE III.}

La quantité de mouvement, qui résulte de la somme de tous les mouvements vers le même côté, et de leurs différences vers des côtés opposés, ne change point par l'action des corps entre eux.

L'action et la réaction sont égales, suivant la troisième loi, donc par la seconde elles produisent dans les mouvements des changements égaux dans des directions opposées. Donc si les mouvements se font du même côté ; ce qui sera ajouté au mouvement du corps chassé, doit être ôté du mouvement de celui qui le suit, en sorte que la somme des mouvements demeure la même qu'auparavant. Si les corps viennent de deux côtés opposés, il faudra retrancher également du mouvement de ces deux corps, et par conséquent la différence des mouvements vers des côtés opposés demeurera toujours la même.

Supposons, par exemple, que la boule $A$ soit triple de la boule $B$, et qu'elle ait deux parties de vitesse, et que $B$ la suive dans la même ligne droite avec 10 parties de vitesse, le mouvement du corps $A$ sera à celui du corps B, comme 6 à 10 : prenant donc 6 et 10 pour exprimer les quantités de mouvement de ces corps, 16 sera la somme de leurs mouvements. 
Lorsque ces corps viendront à se rencontrer, si le corps $A$ gagne 3 , 4 ou 5 parties de mouvement, le corps $B$ en perdra autant, en sorte que le corps $A$, après la réflexion continuant son chemin avec 9,10 ou 11 parties de mouvement, le corps $B$, ira avec 7, 6 ou 5 , et la somme sera toujours de 16 parties comme auparavant. Si le corps $A$ gagne 9,10 , 11 ou 12 parties, et qu'il poursuive par conséquent son chemin après le choc avec 15, 16, 17 ou 18 parties de mouvement ; le corps $B$ perdant tout ce que le corps $A$ gagne, continuera de se mouvoir vers le même côté avec une partie de mouvement, après en avoir perdu 9 , ou il restera en repos, ayant perdu les 10 parties de mouvement progressif qu'il avait, ou il retournera vers le côté opposé avec un degré de mouvement, après avoir perdu tout ce qu'il avait et même une partie de plus (si je puis m'exprimer ainsi), ou bien enfin il retournera vers le côté opposé avec deux parties de mouvement, après avoir perdu 12 parties de son mouvement progressif. Ainsi les sommes des mouvements conspirants $15+1$ ou $16+0$, et les différences des mouvements opposés $17-1$ et $18-2$, feront toujours 16 parties comme avant le choc et la réflexion : connaissant donc la quantité de mouvement avec laquelle les corps se meuvent après la réflexion, on trouvera la vitesse de chacun, en supposant que cette vitesse soit à la vitesse avant la réflexion, comme le mouvement après la réflexion est au mouvement avant la réflexion. Ainsi dans le dernier cas, où le corps $A$ avait 6 parties de mouvement avant la réflexion, et 18 après, et 2 de vitesse avant la réflexion ; on trouverait que la vitesse après la réflexion serait 6 , en disant, comme 6 parties de mouvement avant la réflexion, sont à 18 parties après la réflexion ; ainsi 2 de vitesse avant la réflexion sont à 6 de vitesse après la réflexion.

Si les corps n'étaient pas sphériques, ou que se mouvant suivant diverses lignes droites, ils vinssent à se choquer obliquement, pour trouver leur mouvement après la réflexion ; il faudra commencer par connaître la situation du plan qui touche tous les corps choquants au point de concours : ensuite (par le Cor. 2) on décomposera le mouvement de chaque corps en deux mouvements, l'un perpendiculaire et l'autre parallèle à ce plan tangent : et comme les corps n'agissent les uns sur les autres que selon la ligne perpendiculaire au plan tangent, les mouvements parallèles seront les mêmes après et avant la réflexion ; et les mouvements perpendiculaires éprouveront des changements égaux vers les côtés opposés; en sorte que la somme des 
mouvements conspirants et la différence des mouvements opposés resteront toujours les mêmes qu'auparavant. C'est de ces sortes de réflexions que viennent ordinairement les mouvements circulaires des corps autour de leurs centres; mais je ne considérerai point ces cas dans la suite, parce qu'il serait trop long de démontrer tout ce qui y a rapport.

\section{COROLLAire IV.}

Le centre commun de gravité de deux corps ou de plusieurs corps ne change point son état de mouvement ou de repos, par l'action réciproque de ces corps ; ainsi le centre commun de gravité de tous les corps qui agissent les uns sur les autres (supposé qu'il n'y ait aucune action ni aucun obstacle extérieur) est toujours en repos, ou se meut uniformément en ligne droite.

Car, si deux points se meuvent uniformément en ligne droite, et que leur distance soit divisée en raison donnée, le point de division sera en repos, ou il se mouvra uniformément en ligne droite. C'est ce qu'on trouvera démontré ci-après dans le Lemme 23 et dans son Corollaire, pour le cas où les deux points se meuvent dans le même plan ; et ce qui se démontre facilement par la même méthode pour le cas où les deux points seraient dans des plans différents. Donc, si des corps quelconques se meuvent uniformément en ligne droite, le commun centre de gravité de deux de ces corps, ou sera en repos, ou se mouvra uniformément en ligne droite ; parce que la ligne qui joint les centres de ces corps, sera divisée par leur centre commun de gravité dans une raison donnée. De même le commun centre de gravité de ces deux corps et d'un troisième, sera en repos ou se mouvra uniformément en ligne droite ; à cause que la ligne qui joint le centre commun de gravité de ces deux corps, et le centre du troisième sera encore divisée par le commun centre de gravité de ces trois corps en raison donnée. Enfin le commun centre de gravité de ces trois corps et d'un quatrième quelconque sera, en repos ou sera mû uniformément en ligne droite ; parce que la ligne qui joint le centre commun de gravité de ces trois corps et le centre du quatrième sera divisée par le centre commun de gravité de ces quatre corps en raison donnée et ainsi à l'infini. Donc dans un système de corps, dont les actions réciproques les uns sur les 
autres ne sont point troublées par aucune action ou empêchement externe, et donc par conséquent chacun se meut uniformément en ligne droite, le commun centre de gravité de tous ces corps sera en repos ou sera mû uniformément en ligne droite.

De plus, dans un système composé de deux corps qui agissent l'un sur l'autre, les distances des centres de chacun de ces corps à leur commun centre de gravité étant en raison réciproque de la masse de ces corps ; les mouvements relatifs de ces corps, pour s'éloigner ou pour s’approcher de ce centre commun de gravité, seront égaux entre eux. Donc, ni les changements égaux qui se font dans le mouvement de ces corps en sens contraire, ni par conséquent leur action mutuelle l'un sur l'autre, ne changeront rien à l'état de leur centre commun de gravité qui ne sera ni accéléré ni retardé, et qui ne recevra enfin aucune altération dans son état de mouvement ou de repos.

Puisque dans un système de plusieurs corps, le centre de gravité de deux quelconques de ces corps qui agissent l'un sur l'autre, ne change point d'état par cette action; et que le commun centre de gravité des autres, avec lesquels cette action n'a aucun rapport, n'en souffre aucune altération ; la distance de ces deux centres sera divisée par le centre commun de tous ces corps dans des parties réciproquement proportionnelles aux sommes totales des corps dont ils sont les centres ; et par conséquent ces deux centres conservant leur état de repos ou de mouvement, le centre commun de tous ces corps conservera aussi le sien, car il est clair que le centre commun de tous ces corps ne changera point son état de repos ou de mouvement par les actions de deux quelconques de ces corps entre eux.

Or, dans un tel système, toutes les actions des corps les uns sur les autres, ou sont exercées entre deux corps, ou sont composées d'actions entre deux corps, et par conséquent elles ne produisent aucun changement dans l'état de repos ou de mouvement du centre commun de tous ces corps. C'est pourquoi comme ce centre est en repos, ou qu'il se meut uniformément en ligne droite, lorsque les corps n'agissent point les uns par les autres, il continuera de même, malgré l'action réciproque de ces corps, à être en repos, ou à se mouvoir uniformément en ligne droite, pourvu qu'il ne soit point tiré de son état par des forces étrangères. 
La loi d'un système de plusieurs corps est donc la même que celle d'un corps seul, quant à la permanence dans l'état de repos ou de mouvement uniforme en ligne droite où ils se trouvent. Et le mouvement progressif d'un corps ou d'un système de corps, doit toujours s’estimer par le mouvement de leur centre de gravité.

\section{COROLlaire V.}

Les mouvements des corps enfermés dans un espace quelconque sont les mêmes entre eux, soit que cet espace soit en repos, soit qu'ils se meuvent uniformément en ligne droite sans mouvement circulaire.

Car les différences des mouvements qui tendent vers le même côté, et les sommes de ceux qui tendent vers des côtés opposés, sont les mêmes au commencement du mouvement dans l'un et l'autre cas (par l'hypothèse) mais c'est de ces sommes ou de ces différences qu'on tire l'effort avec lequel les corps se choquent mutuellement : donc par la seconde loi les effets du choc seront les mêmes dans ces deux cas ; et par conséquent les mouvements de ces corps entre eux, dans un de ces cas, demeureront égaux à leurs mouvements entre eux dans l'autre cas, ce que l'expérience confirme tous les jours. Car les mouvements qui se font dans un vaisseau sont les mêmes entre eux, soit que le vaisseau marche uniformément en ligne droite, soit qu'il soit en repos.

\section{COROLLAIRE VI.}

Si des corps se meuvent entre eux d'une façon quelconque, et qu'ils soient poussés par des forces accélératrices égales, et qui agissent sur eux, suivant des lignes parallèles, ils continueront à se mouvoir entre eux de la même manière que si ces forces ne leur avaient pas été imprimées.

Car ces forces agissant également (par rapport à la quantité de matière des corps à mouvoir) et suivant des lignes parallèles, elles feront mouvoir tous ces corps avec des vitesses égales par la seconde loi. Ainsi elles ne changeront point les positions et les mouvements de ces corps entre eux. 


\section{SCHOLIE.}

Les principes que j'ai expliqué jusqu’à présent sont reçus de tous les Mathématiciens, et confirmés par une infinité d'expériences. Les deux premières lois du mouvement et les deux premiers Corollaires ont fait découvrir à Galilée que la descente des graves est en raison doublée du temps, et que les projectiles décrivent une parabole ; ce qui est conforme à l'expérience, si on fait abstraction de la résistance de l'air qui retarde un peu tous ces mouvements.

La gravité étant uniforme, elle agit également à chaque particule égale de temps, ainsi elle imprime au corps qui tombe des vitesses et des forces égales: et dans le temps total elle lui imprime une force totale et une vitesse totale proportionnelle au temps. Mais les espaces décrits dans des temps proportionnels, sont comme les vitesses et les temps conjointement, c'est-à-dire, en raison doublée des temps. Donc, lorsque les corps sont jetés en haut, la gravité leur imprime des forces et leur ôte des vitesses proportionnelles au temps. Ainsi les temps que ces corps mettent à monter à la plus grande hauteur, sont comme les vitesses que la gravité leur fait perdre, et ces hauteurs sont comme les temps multipliés par les vitesses, ou en raison doublée des vitesses. Le mouvement d'un corps jeté suivant une ligne droite quelconque, est donc composé du mouvement de projection et du mouvement que la gravité lui imprime. En sorte que si le corps $A$, par le seul mouvement de projection peut décrire dans un temps donné la droite $A B$, et que par le seul mouvement qui le porte vers la Terre, il puisse décrire la ligne $A C$ dans le même temps: en achevant le parallélogramme $A B C D$, ce corps, par un mouvement composé, sera à la fin de ce temps au lieu $D$; et la courbe $A E D$ qu'il décrira sera une parabole que la droite $A B$ touchera au point $A$, et dont l'ordonnée $B D$ sera proportionnelle à $A B^{2}$.

(Fig. 3)

C’est sur ces mêmes lois et sur leurs corollaires qu'est fondée la théorie des oscillations des Pendules, vérifiée tous les jours par l'expérience. Par ces mêmes lois le Chevalier Christophe Wrenn, J. Wallis S. T. D. et Christian Huygens, qui sont sans contredit les premiers géomètres des derniers temps, ont découvert, chacun de leur côté, les lois du choc et de la réflexion des corps durs ; ils communiquèrent presqu'en même temps leurs découvertes à la Société Royale ; 
ces découvertes s'accordent parfaitement sur ce qui concerne ces lois : Wallis fut le premier qui en fit part à la Société Royale ; ensuite Wrenn, et enfin Huygens ; mais ce fut Wrenn qui les confirma par des expériences faites avec des pendules devant la Société Royale : lesquelles le célèbre Mariotte a rapportées depuis dans un traité qu'il a composé exprès sur cette matière.

(Fig. 4)

Pour que cette théorie s'accorde parfaitement avec l'expérience, il faut faire attention, tant à la résistance de l'air, qu'à la force élastique des corps qui se choquent. Soient $A$ et $B$ des corps sphériques suspendus à des fils parallèles et égaux, $A C, B D$, attachés aux centres $C$ et $D$, et soient décrits autour de ces points comme centre, et des intervalles $A C, B D$, les demi-cercles $E A F, G B H$ séparés chacun en deux parties égales par les rayons $A C, B D$. Si on élève le corps $A$ jusqu'au point quelconque $R$ de l'arc $E A F$, et qu'ayant ôté le corps $B$, on laisse tomber le corps $A$, et que ce corps, après une oscillation, revienne au point $V, R V$ sera le retardement causé par la résistance de l'air. Si on prend alors $S T$ égale à la quatrième partie de $R V$, et placée en telle sorte que $R S=V T, S T$ exprimera à peu près le retardement que le corps $A$ éprouve en descendant de $S$ vers $A$.

Qu'on remette présentement le corps $B$ à sa place, et qu'on laisse tomber le corps $A$, du point $S$, sa vitesse au point $A$ où il doit se réfléchir, sera la même, sans erreur sensible, que s'il tombait du point $T$ dans le vide. Cette vitesse sera donc exprimée par la corde de l'arc $T A$; car c'est une proposition connue de tous les géomètres, que la vitesse d'un corps suspendu par un fil est au point le plus bas de sa chute, comme la corde de l'arc qu'il a parcouru en tombant.

Supposons que le corps $A$ parvienne après la réflexion en $s$, et le corps $B$ en $k$, qu'on ôte encore le corps $B$, et qu'on trouve le lieu $v$ duquel laissant tomber le corps $A$, ils reviennent après une oscillation au lieu $r$, de plus que st soit la quatrième partie de $r v$ placée en telle sorte que $r s=t v$, $t A$ exprimera la vitesse que le corps $A$ avait en $A$ l'instant d'après la réflexion. Car $t$ sera le lieu vrai et corrigé auquel le corps $A$ devrait remonter, si l'on faisait abstraction de la résistance de l'air. On corrigera par la même méthode le lieu $k$, auquel le corps $B$ remonte, et on trouvera le lieu $l$ auquel il aurait dû remonter dans le vide, et par ce moyen on fera ces expériences aussi exactement dans l'air que dans le 
vide. Enfin pour avoir le mouvement du corps $A$, au lieu $A$, immédiatement avant la réflexion, il faudra multiplier le corps $A$, si je puis m'exprimer ainsi, par la corde de l'arc $T A$, qui exprime sa vitesse ; ensuite il faut le multiplier par la corde de l'arc $t A$, pour avoir son mouvement au lieu $A$, immédiatement après la réflexion. De même, il faudra multiplier le corps $B$, par la corde de l'arc $B l$, pour avoir son mouvement immédiatement après la réflexion.

Par la même méthode, lorsque les deux corps tomberont en même temps de deux hauteurs différentes, on trouvera le mouvement de l'un et de l'autre, tant avant qu'après la réflexion ; et l'on pourra toujours, par ce moyen, comparer ces mouvements entre eux, et en conclure les effets de la réflexion.

Suivant cette méthode, dans les expériences que j'ai faites avec des Pendules de 10 pieds de long auxquels j'avais suspendu tantôt des corps égaux, tantôt des corps inégaux, et que j'avais fait se choquer en tombant de très haut, comme de 8,12 et 16 pieds, j'ai toujours trouvé, à des différences près, lesquelles étaient moindres que trois pouces dans les mesures, que lorsque les corps se rencontraient directement, les changements de mouvement vers les points opposés étaient toujours égaux, et que par conséquent la réaction était toujours égale à l'action. Lorsque le corps $A$, par exemple, ayant 9 parties de mouvement venait à choquer le corps $B$ en repos, et qu'après avoir perdu 7 parties de mouvement, il continuait après la réflexion à se mouvoir avec deux parties, le corps $B$ rejaillissait avec ces 7 parties.

Si les deux corps allaient l'un vers l'autre, $A$ avec 12 parties de mouvement et $B$ avec 6 , et qu'après le choc $A$ s'en retournât avec 2 parties, $B$ s'en retournait avec 8 , et il y avait 14 parties de détruites de chaque côté. Car si du mouvement de $A$ on en ôte d'abord 12 parties, il ne lui reste rien : si on ôte ensuite 2 autres parties, il en naît deux parties de mouvement en sens contraire : de même en ôtant 14 parties du mouvement du corps $B$, il en naît 8 parties vers le côté opposé.

Lorsque les deux corps allaient vers le même coté, $A$ plus vite avec 14 parties de mouvement, et $B$ plus lentement avec 5 parties, et qu'après la réflexion le corps $A$ continuait de se mouvoir avec 5 parties, le corps $B$ continuait alors à se mouvoir avec 14 parties, en sorte qu'il avait acquis les neuf parties que le corps $A$ avait perdu; il en 
était de même dans tous les autres cas. La quantité de mouvement n'était jamais changée par le choc, elle se retrouvait toujours ou dans la somme des mouvements conspirants ou dans la différence des mouvements opposés ; et j'ai attribué les erreurs d'un ou deux pouces que j'ai trouvées dans les mesures à la difficulté de prendre ces mesures avec assez d'exactitude; car il était difficile de faire tomber les pendules dans le même instant, en sorte que les corps se rencontrassent dans le lieu le plus bas $A B$; et de marquer exactement les lieux $S$ et $k$ auxquels les corps remontaient après le choc; et il pouvait encore s'y mêler d'autres causes d'erreur, comme l'inégale densité des parties des corps suspendus, leur différente texture, etc.

Et afin qu'on ne m'objecte pas que la loi que j'ai voulu prouver par ces Expériences suppose les corps ou parfaitement durs, ou parfaitement élastiques, et que nous ne connaissons point de tels corps, j'ajouterai que ces expériences réussissent aussi bien sur les corps mous que sur les corps durs, et que par conséquent la vérité de ce principe ne dépend point de la dureté des corps; car si on veut l'appliquer aux cas où les corps ne sont pas parfaitement durs, il faudra seulement diminuer la réflexion dans une certaine proportion relative à la quantité de la force élastique.

Dans la théorie de Wrenn et d'Huygens, les corps absolument durs, après s'être choqués, s'éloignent l'un de l'autre avec la même vitesse qu'ils avaient dans le choc. On peut l'assurer avec encore plus de certitude des corps parfaitement élastiques. Dans les corps qui ne sont pas parfaitement élastiques, la vitesse avec laquelle ils s'en retournent après le choc, doit être diminuée relativement à la force élastique ; et parce que cette force (pourvu que les parties des corps ne soient point altérées par la collision, ou qu'elles ne souffrent point d'extension comme celle que cause le marteau) est constante et déterminée, ainsi que je l'ai remarqué ; elle fait que les corps rejaillissent avec une vitesse relative qui est à la vitesse qu'ils avaient avant le choc dans une raison donnée.

Je fis aussi cette expérience avec des pelotes de laine très serrées. Je commençai par déterminer la quantité de la force élastique, en faisant tomber les Pendules et en mesurant la réflexion : et ensuite connaissant cette force, j'en conclus les réflexions pour d'autres cas, et je trouvai que les expériences y répondaient. Les pelotes 
s'éloignaient toujours l'une de l'autre après le choc avec une vitesse relative, qui était à leur vitesse relative dans le choc, comme 5 à 9 environ. Les boules d'acier rejaillissaient à peu près avec leur même vitesse : les boules de liège rejaillissaient avec une vitesse un peu moindre ; et dans les boules de verre ces vitesses étaient à peu près comme 15 à 16. Ainsi la troisième loi trouve confirmée dans le choc et dans la réflexion des corps par la théorie, et la théorie l'est par l'expérience. Je vais faire voir qu'elle l'est aussi dans les attractions.

Imaginez entre les deux corps $A$ et $B$ un obstacle quelconque qui les empêche de se joindre. Si un de ces corps comme $A$ est plus attiré vers $B$, que $B$ vers $A$, l'obstacle sera plus pressé par le corps $A$ que par le corps $B$; ainsi il ne sera point en équilibre. La plus forte pression prévaudra, et il arrivera que le système, composé de ces deux corps et de l'obstacle qui est entre deux, se mouvra en ligne droite vers $B$, et qu'il s'en ira à l'infini, dans le vide avec un mouvement continuellement accéléré, ce qui est absurde et contraire à la première loi du mouvement ; car par cette première loi, ce système doit persévérer dans son état de repos ou de mouvement en ligne droite, ainsi ces deux corps doivent presser également cet obstacle, et être par conséquent tirés également l'un vers l'autre.

J'en ai fait l'expérience sur le fer et sur l'aimant. Si l'on pose l'aimant et le fer chacun séparément dans de petits vaisseaux sur une eau dormante, et que ces petits vaisseaux se touchent, ni l'un ni l'autre ne sera mû ; mais ils soutiendront par l'égalité de leur attraction les efforts mutuels qu'ils font l'un sur l'autre, et étant en équilibre, ils resteront en repos.

(Fig.5)

De même, la gravité entre la Terre et ses parties est mutuelle ; car supposé que la Terre FI fût coupée par un plan EG en deux parties $E G F, E G I$ : les poids mutuels de ces parties l'une sur l'autre, seront égaux ; car si la plus grande partie EGI est coupée par un autre plan $H K$ parallèle au premier, en deux parties $E G H K$ et $H I K$, desquelles $H I K=E F G$ : il est clair que la partie du milieu EGHK ne sera, portée par son propre poids ni vers l'une, ni vers l'autre de ces parties, mais qu'elle restera en équilibre entre elles. 
Quant à la partie $H I K$, elle pressera de tout son poids la partie du milieu vers l'autre partie $E F G$; donc la force avec laquelle la partie $E G I$, composée des parties $H K I$ et $E G H K$, tend vers la troisième partie $E F G$, est égale au poids de la partie $H I K$, c'est-à-dire au poids de la troisième partie $E F G$. Ainsi le poids de deux parties EGI, EFG, l'une sur l'autre est égal, ce que je voulais prouver. Et si ces poids n'étaient pas égaux, toute la Terre qui nage librement dans l'éther céderait au plus grand de ces poids, et s'en irait à l'infini.

De même que les corps qui se choquent se sont équilibrés, quand leurs vitesses sont réciproquement comme leurs forces d'inertie (ut vires infitae) les puissances qui agissent dans la mécanique se contrebalancent et détruisent leurs efforts mutuels, quand leurs vitesses dans la direction des forces sont réciproquement comme ces forces. Ainsi des poids attachés aux bras d'une balance font des efforts égaux pour la mouvoir, lorsque ces poids sont réciproquement comme les vitesses qu'auraient les bras de la balance en haut et en bas. Si elle venait à osciller ; c'est-à-dire, que ces poids sont en équilibre, lorsque les bras de la balance montent et descendent perpendiculairement, s'ils sont entre eux réciproquement comme la distance du point de suspension au fléau de la balance ; et si les bras de la balance montent et descendent obliquement, soit qu'ils soient soutenus par des plans obliques, ou que quelque autre obstacle les empêche de monter et de descendre perpendiculairement, les poids seront en équilibre, lorsqu'ils seront entre eux réciproquement, comme l'ascension et la descension perpendiculaire des bras de la balance; parce que la force de la gravité est toujours dirigée perpendiculairement vers la Terre.

De même, dans la poulie ou dans le mouffle, si la force de la main qui tire la corde directement, est au poids qui monte directement ou obliquement, comme la vitesse de son ascension perpendiculaire à la vitesse de la main qui tire la corde, il y aura équilibre.

Dans les Horloges et les autres machines, dont la construction dépend du jeu de plusieurs roues, les forces contraires qui font des efforts pour les mouvoir et pour les retenir, se contrebalanceront mutuellement, si elles sont entre elles réciproquement comme les vitesses des parties des roues auxquelles elles sont imprimées. 
La force de la vis pour presser un corps est à la force de la main qui tourne la manivelle, comme la vitesse circulaire de la manivelle dans la partie où la main la fait tourner, est à la vitesse progressive de la vis vers le corps qu'elle presse.

Les forces avec lesquelles le coin presse les deux côtés du bois qu'il fend, sont à la force avec laquelle le marteau frappe le coin, comme le chemin que fait le coin dans la direction de la force que lui impriment les coups du marteau, est à la vitesse avec laquelle les parties du bois cèdent au coin selon les lignes perpendiculaires aux faces du coin. Il en est de même dans toutes les machines dont l'efficacité consiste en cela, seulement, qu'en diminuant la vitesse on augmente la force et réciproquement; et c'est par-là qu'on résout ce problème dans toutes les espèces de machines, que le poids étant donné, la force nécessaire pour le mouvoir est donnée, ou ce qui est la même chose, que la résistance étant donnée, la force nécessaire pour la surmonter est donnée aussi. Car lorsque les machines seront construites de façon que la vitesse de la puissance soit à celle de la résistance en raison renversée des forces ; la puissance égalera la résistance : et si on augmente la vitesse de la puissance, elle vaincra aussitôt la résistance.

Si la disparité des vitesses est assez grande pour vaincre toute espèce de résistance, tant celle qu'oppose la pesanteur des corps qu'on veut élever, que celle qui vient de la cohésion des corps qu'on veut séparer, et que celle qui est produite par le frottement des corps qui glissent les uns sur les autres, la force restante produira une accélération de mouvement qui lui sera proportionnelle, et qui sera partagée entre les parties de la machine, et le corps résistant; mais je ne me suis pas proposé ici de donner un Traité de Mécanique, j’ai voulu montrer seulement combien la troisième loi du mouvement est vraie, et combien son usage est étendu, car si on estime l'action de l'agent par sa force multipliée par sa vitesse et qu'on estime de même la réaction du corps résistant par la vitesse de chacune de ces parties multipliées par les forces qu'elles ont pour résister en vertu de leur cohésion, de leur attrition, de leur poids, et de leur accélération, l'action et les réactions se trouveront égales entre elles, dans les effets de toutes les machines. Et toutes les fois qu'une action s'exécute par le moyen d'une machine, et qu'elle parvient à être imprimée dans un corps résistant, sa dernière détermination est toujours contraire à la détermination de la réaction de ce corps. 
